\title{
A new Savage-Hutter type model for submarine avalanches and generated tsunami
}

\author{
E.D. Fernández-Nieto, $\stackrel{*}{, F}$. Bouchut $\stackrel{\dagger}{\text { D. Bresch }} ¥$ \\ M.J. Castro Díaz $\stackrel{\&}{\&}$ A. Mangeney $₫$
}

\begin{abstract}
In this paper we present a new two-layer model of Savage-Hutter type to study submarine avalanches. A layer composed of fluidized granular material is assumed to flow within an upper layer composed of an inviscid fluid (e. g. water). The model is derived in a system of local coordinates following a non-erodible bottom and takes into account its curvature. We prove that the model verifies an entropy inequality, preserves water at rest for a sediment layer and their solutions can be seen as particular solutions of incompressible Euler equations under hydrostatic assumptions. Buoyancy effects and the centripetal acceleration of the grain movement due to the curvature of the bottom are considered in the definition of the Coulomb term. We propose a two-step Roe type solver to discretize the presented model. It exactly preserves water at rest and no movement of the sediment layer, when its angle is smaller than the angle of repose, and up to second order all stationary solutions. Finally, some numerical tests are performed by simulating submarine and sub-aerial avalanches as well as the generated tsunami.
\end{abstract}

\section{Introduction}

Recent improvements in seabed and sub-surface mapping techniques as bathymetry measurements and seismic imagery, have revealed a large amount of slide scars and a wide diversity of related deposits on many of the world's continental margins [e. g. Locat and Mienert, 2003, Vanneste et al., 2006]. Submarine avalanches or landslides are poorly studied compared to their subaerial counterparts. This is however a key issue in geophysics. Indeed, submarine granular flows driven by gravity participate in the evolution of the sea floor and in particular of the continental margins. They also represent a threat to the submarine infrastructures, especially for the oil or port industry as well as to many sea shore inhabitants due to the potential tsunamis that can be triggered by such landslides. In this paper we present a new two-layer Savage-Hutter type model, with application to sub-aerial/submarine avalanches over variable topography and generated tsunami. The first layer is filled with a homogenous inviscid fluid with constant density and the second layer is made of a fluidized granular mass. The two fluids (i. e. water and fluidized debris) are assumed to be immiscible. Important questions are $(i)$ the rheological behavior of the fluidized granular mass on a complex topography and $(i i)$ the interaction between the two layers.

\footnotetext{
*Departamento de Matemática Aplicada I, Universidad de Sevilla. E.T.S. Arquitectura. Avda, Reina Mercedes, s/n. 41012 Sevilla, Spain (edofer@us.es)

†Département de mathématiques et applications, CNRS \& École normale supérieure, 45, rue d’Ulm, 75230 Paris cedex 05, France (fbouchut@dma.ens.fr)

${ }^{\ddagger}$ Laboratoire de Mathématiques, UMR 5127 CNRS, Univ. Savoie, 73376 Le Bourget du Lac (France) (Didier.Bresch@univ-savoie.fr)

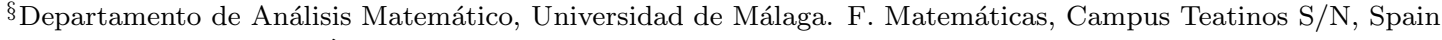
(castro@anamat.cie.uma.es)

IEquipe de Sismologie, IPGP, 4, pl. Jussieu, 75232 Paris cedes 05, France and Institute for Nonlinear Science, University of California San Diego, 9500 Gilman Drive, La Jolla, CA 92093-0402, USA (mangeney@ipgp.jussieu.fr)
} 
Numerical modeling of sub-aerial debris or snow avalanches has been extensively investigated during this last decade with application to both laboratory experiments dealing with granular flows and geological events (see e. g. [32], [29], [39], [49], [21], [2], [3], [27], [5], [1]). Most of the models devoted to gravitational granular flows describe the behavior of dry granular material following the pioneer work of Savage and Hutter (see [45]): a shallow-water type model (i. e. thin layer approximation for a continuum medium) is derived to describe granular flows over a slopping plane based on Mohr-Coulomb considerations: a Coulomb friction is assumed to reflect the avalanche/bottom interaction and the normal stress tensor is defined by a constitutive law relating the longitudinal and the normal stresses through a proportionality factor $K$.

New Savage-Hutter models over a general bottom have been proposed by Bouchut et al. in [6], that take into account the curvature of the bottom. The authors introduce two new models: the first one is deduced under the hypothesis of small variation of the curvature and the second one deals with a general bottom topography. The new curvature terms introduced in the models are necessary for two reasons: they make it possible to preserve water at rest solutions and to exactly verify an energy inequality. In this paper we consider the first hypothesis, i. e. a small variation of the curvature. The equations are derived in a local coordinate system attached to the non-erodible topography and takes into account its curvature (see [6]), in particular the centripetal acceleration due to the bottom curvature.

A generalization to 2D aerial avalanches over surfaces with small lateral curvature has been carried out in [49] and [41]. In [7], Bouchut and Westdickenberg generalize the previous models for small or for general slope variation in two dimensions. The discretization of $2 \mathrm{D}$ aerial avalanches can be done for example by finite volume by using kinetic schemes [31], by Roe type finite volume methods [11], or distribution schemes [43].

A two-layer Shallow Water type model with compressible effects has been introduced in [34] by Morales de Luna. He considers an upper compressible and a lower incompressible layer. The model is presented in local coordinates, verifies an entropy dissipation inequality and gives an approximation of the free surface compressible-incompressible Euler equations.

In most industrial applications and real debris flows, the fluid which is present in the granular material cannot be neglected. Recent attempts have been developed to describe mixtures of grains and fluids in shallow-water two-phase or mixture models ([26], [40], [37], [42]). Iverson and Denlinger extend the SH model in [26] to study avalanches of fluidized granular masses where the pores between the grains are assumed to be filled with a fluid. In [42], Pudasaini, Wang and Hutter generalized the work [26] for a general channel on local coordinates. In both works a simplified system is considered, assuming that the velocity of the fluid within the pores is equal to the velocity of the grains. The same hypothesis is used here: the fluidized mass is assumed to be a porous medium composed of sand grains, filled with the fluid present in the upper layer (see [26]). The dissipation within the granular medium is modeled by a Coulomb friction law taking into account the buoyancy effects over the sand grains. The other key point concerns the definition of the stress tensor for the fluid and grain phases of the second layer. From the vertical momentum equation and dimensional analysis, the vertical stress tensor of the complete layer can be derived. However, it is necessary to know the stress tensor for each phase (i. e. fluid and solid phase) in order to apply different constitutive relations for the fluid and solid phase separately. Therefore, additional hypotheses have to be introduced (see [26]).

Finally, very few models have been proposed to deal with the interaction of a fluidized mass and the surrounding fluid in which the avalanche propagates. One of the outcome of the interaction between water and debris is the generation of water waves and possible tsunami for particular configurations of the coastal topography and of the submarine avalanche. Most of the models dedicated to the simulation of landslide generated tsunamis reduce the trigger mechanism to a vertical motion imposed as boundary condition in the water wave propagation model (see for example [20]). Submarine landslides are actually modeled by partially or totally submerged pistons, rigid bodies entering the water or initial water displacement (see e. g. [44], [35]). More recently, submarine avalanche dynamics has been taken into account using depth-averaged or full Navier-Stokes models 
describing the rheological behavior by a Coulomb friction law or by viscous dissipation ([22], [23], [30], [19]). A similar attempt has been performed by Heinrich et al. in [24] but without taking into account the effects of the fluid on the landslide dynamics (i. e. the sea-bottom deformation induced by the landslide is used as input data in the tsunami model). As a result, the momentum equation for the fluidized granular material does not contain any coupling terms between the two layers which should appear in the pressure gradient terms. Other systems, named active models, with a dynamic displacement of sea bed are used with a coupling between a shallow-water system and visco-elastic equations, see for instance [16], [17]. The interested reader is referred to [15] and [18] for references around Tsunamis and challenging modeling.

In this paper we present a 1D model for submarine avalanches, which is a generalization of the Savage Hutter (SH) 1D model [45] for aerial avalanches and the model proposed by Heinrich et al. in [24].

To discretize the model that we introduce in the paper, we propose a well-balanced finite volume method. Firstly we begin by rewriting the model obtained in local coordinates to Cartesian coordinates. We can write the model as a hyperbolic system with conservative terms, source terms and non-conservative terms. One of the characteristics of the model is that as we consider the variations of the topography, the physical flux function depends on the variable $x$ measured along the horizontal coordinate. This dependence of the flux with respect to $x$ makes difficult the derivation of an exact well-balanced method for water at rest (see [9], [36]). Moreover, for the proposed model the water at at rest solution should be understood as: no movement of the water column and no movement of the sediment layer when the angle of the sediment surface is smaller than the angle of repose. In such situations, it is necessary to discretize properly the source terms due to the variations of the bottom angle and the derivatives of the flux function with respect to the angle.

The more specific difficulty related to discretizisation of system comes from the Coulomb friction term. Its discretization is important, to simulate properly the landslides and to preserve the stationary solutions corresponding to water at rest and no movement of the sediment layer. We propose a two-step numerical scheme to treat the Coulomb friction term. In a first step, a discretization of a term that can be interpreted as a redefinition of the Coulomb term for stationary solutions is considered. This term is only introduced in the uncentered component of the numerical scheme. In the second step, a semi-implicit treatment of the Coulomb term at each cell is performed. We proof that the numerical scheme constructed in this way preserves the solutions corresponding to water at rest and no movement of the sediment layer for angles smaller than the angle of repose.

The paper is organized as follows: the model is derived in Section 2. Section 3 is devoted to study the model properties. In Section 4 we present a well-balanced finite volume numerical scheme to discretize the model. We proof that the numerical scheme exactly preserves water at rest and no movement of the sediment layer, and up to second order all stationary solutions. Finally, in Section 5 a series of numerical tests are performed, including the simulation of a tsunami generated by the motion of a sediment layer, following [24]. In Appendix A we present the details related to the change of variable used in the incrompressible Euler equations to write the equation in a local coordinate system attached to the bottom topography.

\section{Derivation of the model}

In this section, we present the derivation of a two-layer model of Savage-Hutter type to study submarine avalanches and generated tsunamis. We denote with index 1 the upper layer, composed of a homogeneous inviscid fluid of constant density $\rho_{1}$. We also consider a grain layer of density $\rho_{s}$, and porosity $\psi_{0}$ (see Figure 1). We consider that the pores in the grain layer are filled with the 


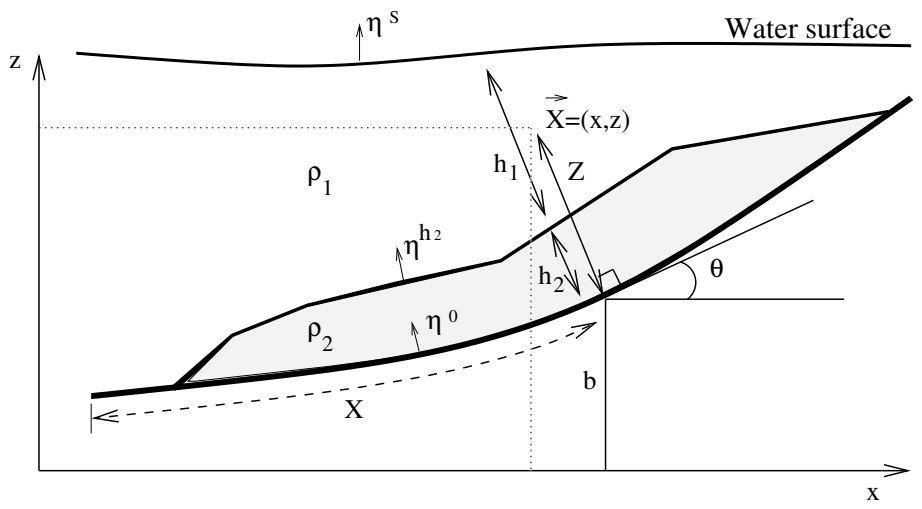

Figure 1: A fluid layer over a grain layer and a non-erodible bottom $b$

fluid of the upper layer. Then, the density of layer 2 composed of the fluidized mass is defined as

$$
\rho_{2}=\left(1-\psi_{0}\right) \rho_{s}+\psi_{0} \rho_{1}
$$

First, the system of equations describing the dynamics of the two-layer system is presented. Next, a change of variables to local coordinates attached to the bottom (see Appendix) is performed and the boundary and kinematic conditions are set. The final model is derived based on a dimensional analysis and a vertical integration of the equations.

\section{Starting system of equations}

We consider the incompressible Euler equations. The unknowns are

$$
\vec{V}_{i}=\left(\begin{array}{c}
u_{i} \\
v_{i}
\end{array}\right), \quad i=1,2
$$

being $u_{i}$ and $v_{i}$, the horizontal and vertical velocity components of each layer, respectively. Then, the incompressible Euler equations can be written as

$$
\begin{gathered}
\operatorname{div} \vec{V}_{i}=0, i=1,2, \\
\rho_{i} \partial_{t} \vec{V}_{i}+\rho_{i} \vec{V}_{i} \nabla \vec{V}_{i}=-\operatorname{div} P_{i}+\rho_{i} \nabla(\vec{g} \cdot \vec{X}), \quad i=1,2,
\end{gathered}
$$

where we denote by $P_{i}, i=1,2$, the pressure tensor of each layer

$$
P_{i}=\left(\begin{array}{cc}
p_{i, x x} & p_{i, x z} \\
p_{i, z x} & p_{i, z z}
\end{array}\right), i=1,2,
$$

(with $p_{i, x z}=p_{i, z x}$ ), by $\rho_{i}, i=1,2$ the densities of each layer, by $\vec{X}$ a point in Cartesian coordinates $\vec{X}=(x, z)$, and $\vec{g}=(0,-g)$.

In order to model the evolution of the granular layer using the Euler equations, following [26] we suppose that the velocity of the fluid in the pores of the second layer and the grains are the same and $P_{2}$ can be decomposed as

$$
P_{2}=P_{2}^{s}+P_{2}^{f},
$$

where $P_{2}^{s}$ and $P_{2}^{f}$ are the pressure tensor of the solid phase (grains) and the fluid phase, ${ }^{1}$ respectively.

\footnotetext{
${ }^{1}$ In a binary mixture model the pressure tensor of the mixture is exactly given by

$$
P=P^{s}+P^{f}-\sum_{\alpha=1}^{2} \rho_{\alpha}\left(\vec{V}_{\alpha}-\vec{V}_{b}\right) \otimes\left(\vec{V}_{\alpha}-\vec{V}_{b}\right), \quad \text { where } \quad \vec{V}_{b}=\sum_{\alpha=1}^{2} \frac{\rho_{\alpha} \vec{V}_{\alpha}}{\sum_{\alpha=1}^{2} \rho_{\alpha}}
$$

is the barycentric velocity. This reduces to $P=P^{s}+P^{f}$ if the fluid and solid velocities are the same.
} 
Next, a change of variables is performed to equations (2)-(3). Local variables over a nonerodible bottom defined by $z=b(x)$ are considered. $X$ denotes the arc's length of the bottom and $Z$ is measured orthogonally to the bottom (see Figure 1 ).

In what follows we denote by $h_{1}$ and $h_{2}$ the thickness of the fluid and grain layers, respectively, measured orthogonally to the bottom (see Figure 1), by $S=h_{1}+h_{2}$ the free water surface.

The details of this change of variables is given in the Appendix. The change of variables is valid when the local radius of curvature of the bed is smaller than $h_{1}+h_{2}$. Equations (2)-(3) are re-written in the new variables as

$$
\left\{\begin{array}{l}
\partial_{X}\left(U_{i}\right)+\partial_{Z}\left(J W_{i}\right)=0, \quad i=1,2 \\
\rho_{i} \partial_{t}\left(J U_{i}\right)+\rho_{i} \partial_{X}\left(U_{i}^{2}\right)+\rho_{i} \partial_{Z}\left(J W_{i} U_{i}\right)+\rho_{i} \partial_{X}(\vec{g} \cdot \vec{X})=-\partial_{X}\left(\mathcal{P}_{i X X}\right)-\partial_{Z}\left(J \mathcal{P}_{i Z X}\right)+ \\
+\rho_{i} W_{i}\left(\partial_{X}\left(U_{i} \theta\right)+\partial_{Z}\left(J W_{i} \theta\right)\right)+\mathcal{P}_{i X Z} d_{X} \theta . \quad i=1,2 \\
\rho_{i} \partial_{t}\left(J W_{i}\right)+\rho_{i} \partial_{X}\left(U_{i} W_{i}\right)+\rho_{i} \partial_{Z}\left(J W_{i}^{2}\right)+\rho_{i} J \partial_{Z}(\vec{g} \vec{X})=-\partial_{X}\left(\mathcal{P}_{i X Z}\right)- \\
-\partial_{Z}\left(J \mathcal{P}_{i Z Z}\right)-\rho_{i} U_{i}\left(\partial_{X}\left(U_{i} \theta\right)+\partial_{Z}\left(J W_{i} \theta\right)\right)-\mathcal{P}_{i X X} d_{X} \theta, \quad i=1,2,
\end{array}\right.
$$

where, we denote by $U_{i}, i=1,2$, the velocity parallel to the bottom and by $W_{i}, i=1,2$, the velocity perpendicular to the bottom, with $i$ referring to layers 1 and 2 . The pressure tensor $\mathcal{P}_{i}$ is defined by

$$
\mathcal{P}_{i}=\left(\begin{array}{cc}
\cos \theta & \sin \theta \\
-\sin \theta & \cos \theta
\end{array}\right) P_{i}\left(\begin{array}{cc}
\cos \theta & -\sin \theta \\
\sin \theta & \cos \theta
\end{array}\right)=\left(\begin{array}{cc}
\mathcal{P}_{i, X X} & \mathcal{P}_{i, X Z} \\
\mathcal{P}_{i, Z X} & \mathcal{P}_{i, Z Z}
\end{array}\right)
$$

Observe that as $p_{i, x z}=p_{i, x z}$ then $\mathcal{P}_{i, X Z}=\mathcal{P}_{i, Z X}$.

Moreover, let us recall that $\rho_{1}$ is the density of the fluid and that $\rho_{2}$ is defined by (1). $\theta$ is the angle between the tangent vector of the bottom and the horizontal (see Figure 1), and $J=1-Z d_{X} \theta$ is the Jacobian of the change of variables (note, $d_{X} \theta=\partial_{X} \theta$, for a non-erodible bed, see Appendix). Observe that $J \neq 0$ if the local radius of curvature of the bed is smaller than $h_{1}+h_{2}$.

\section{Boundary and kinematic conditions}

We denote by $\eta^{S}$ the unitary normal vector to the free water surface $Z=S\left(S=h_{1}+h_{2}\right)$ with positive vertical component, by $\eta^{h_{2}}$ the unitary normal vector to the surface $Z=h_{2}$ and by $\eta^{0}=(0,1)$ the corresponding unitary normal vector to the bottom $(Z=0)$.

The following kinematic conditions are considered

$$
\begin{gathered}
\partial_{t} S+U_{1} \partial_{X} S-W_{1}=0 \\
\partial_{t} h_{2}+U_{i} \partial_{X} h_{2}-W_{i}=0, \quad i=1,2 .
\end{gathered}
$$

Finally, the following boundary conditions are imposed:

- On $\mathbf{Z}=S$ :

$$
\mathcal{P}_{1} \cdot \eta^{S}=0
$$

- On $\mathbf{Z}=h_{2}$ :

$$
\begin{gathered}
\eta^{h_{2}} \cdot\left(\mathcal{P}_{1}-\mathcal{P}_{2}\right) \eta^{h_{2}}=0 \\
\mathcal{P}_{i} \cdot \eta^{h_{2}}-\eta^{h_{2}}\left(\eta^{h_{2}} \cdot \mathcal{P}_{i} \eta^{h_{2}}\right)=\left(\begin{array}{c}
\operatorname{fric}\left(U_{1}, U_{2}\right) \\
0
\end{array}\right) \quad i=1,2,
\end{gathered}
$$

where $\operatorname{fric}\left(U_{1}, U_{2}\right)$ is a friction term between both layers. 
- On $\mathbf{Z}=0$ :

$$
\begin{gathered}
(U, W) \cdot \eta^{0}=0 \quad \Rightarrow \quad W=0 \\
\mathcal{P}_{2} \eta^{0}-\eta^{0}\left(\eta^{0} \cdot \mathcal{P}_{2} \eta^{0}\right)=\left(\begin{array}{c}
-\eta^{0} \cdot\left(\mathcal{P}_{2}-\mathcal{P}_{1}\right) \eta^{0} \frac{U_{2}^{0}}{\left|U_{2}^{0}\right|} \tan \left(\delta_{0}\right) \\
0
\end{array}\right) .
\end{gathered}
$$

Note that the Coulomb friction law in equation (11) takes into account the buoyancy effects due to the fact that the grains are submerged within a fluid layer.

Remark 1 Equation (9) assumes no water exchange between the two layers. Nevertheless there is a water exchange between the fluid an the porous avalanche, so equation (9) constitutes a simplification of the problem. This entrainment process has been studied first by Beaves and Joseph in [4].

Equation (6) assumes that the second layer has constant porosity (volume fraction) since $\rho_{2}$ is constant then $\psi_{0}=$ constant.

\section{Dimensional analysis}

Next, a dimensional analysis of the set of equations (4), the kinematic and boundary conditions is performed. The non-dimensional variables $(\sim)$ read:

$$
\begin{aligned}
& (X, Z, t)=\left(L \widetilde{X}, H \widetilde{Z},(L / g)^{1 / 2} \widetilde{t}\right), \\
& \left(U_{i}, W_{i}\right)=(L g)^{1 / 2}\left(\widetilde{U}_{i}, \varepsilon W_{i}\right), i=1,2, \\
& h_{i}=H \widetilde{h}_{i}, i=1,2, \quad\left(\mathcal{P}_{i X X}, \mathcal{P}_{i Z Z}\right)=g H\left(\widetilde{\mathcal{P}}_{i X X}, \widetilde{\mathcal{P}}_{i Z Z}\right), i=1,2, \\
& \mathcal{P}_{i X Z}=g H \mu_{i} \widetilde{\mathcal{P}}_{i X Z}, \quad i=1,2,
\end{aligned}
$$

where $\mu_{1}=1, \mu_{2}=\tan \left(\delta_{0}\right), \delta_{0}$ being the angle of repose in the Coulomb term (see [45]). By $L$ and $H$ we denote, respectively, the characteristic lengths tangential and normal to a representative basal direction of the domain. We suppose a shallow domain, so $\varepsilon=H / L$ is supposed to be small. Note that the Savage Hutter model has been shown to reproduce experimental granular collapse over horizontal plane for aspect ratio $\epsilon \leq 0.5$ [32]. Using the above change of variables, the system of equations (4) are re-written as (we omit the tildes):

$$
\begin{gathered}
\partial_{X}\left(U_{i}\right)+\partial_{Z}\left(J W_{i}\right)=0, \quad i=1,2, \\
J \partial_{t}\left(\rho_{i} U_{i}\right)+\rho_{i} U_{i} \partial_{X} U_{i}+\rho_{i} J W_{i} \partial_{Z} U_{i}+\rho_{i} \partial_{X}\left(b+Z \cos \theta+\frac{\mathcal{P}_{i X X}}{\rho_{i}}\right) \varepsilon= \\
=-\mu_{i} \partial_{Z}\left(J \mathcal{P}_{i X Z}\right)+\rho_{i} W_{i} \varepsilon U_{i} d_{X} \theta+\partial_{X} \theta \mathcal{P}_{i X Z} \mu_{i} \varepsilon, i=1,2, \\
\varepsilon\left\{J \partial_{t}\left(\rho_{i} W_{i}\right)+\rho_{i} U_{i} \partial_{X}\left(W_{i}\right)+\rho_{i} W_{i} \partial_{Z}\left(W_{i}\right)+\partial_{X}\left(\mathcal{P}_{i X Z}\right)-\partial_{X} \theta \mathcal{P}_{i X X}-\mathcal{P}_{i Z Z} d_{X} \theta\right\}+ \\
+\rho_{i} J \partial_{Z}(b+\cos \theta Z)=-J \partial_{Z}\left(\mathcal{P}_{i Z Z}\right)-\rho_{i} U_{i}^{2} d_{X} \theta, \quad i=1,2 .
\end{gathered}
$$

The kinematic conditions (5)-(6) are re-written as:

$$
\partial_{t} S+U_{1} \partial_{X} S-W_{1}=0, \quad \partial_{t} h_{2}+U_{i} \partial_{X} h_{2}-W_{i}=0, \quad i=1,2 .
$$

Finally, the boundary conditions (7)-(11) are now given as: 
- $\mathbf{Z}=S:$ On $Z=S$ we have, $\eta^{S}=\left(-\varepsilon \partial_{X} S, 1\right) / \varphi^{S}$ with $\varphi^{S}=\sqrt{1+\varepsilon^{2}\left(\partial_{X} S\right)^{2}}$, then from $(7)$ we obtain

$$
\begin{aligned}
& -\varepsilon \partial_{X} S \mathcal{P}_{1 X X}+\mu_{1} \mathcal{P}_{1 Z X}=0 \\
& -\varepsilon \partial_{X} S \mu_{1} \mathcal{P}_{1 X Z}+\mathcal{P}_{1 Z Z}=0 .
\end{aligned}
$$

- $\mathbf{Z}=h_{2}$ : On $Z=h_{2}$ we have, $\eta^{h_{2}}=\left(-\varepsilon \partial_{X} h_{2}, 1\right) / \varphi^{h_{2}}$ with $\varphi^{h_{2}}=\sqrt{1+\varepsilon^{2}\left(\partial_{X} h_{2}\right)^{2}}$, then from (8) and (9) we obtain

$$
\begin{gathered}
\mathcal{P}_{1 Z Z}=\mathcal{P}_{2 Z Z}+\mathcal{O}(\varepsilon) \\
-\varepsilon \mathcal{P}_{i X X} \partial_{X} h_{2}+\mu_{i} \mathcal{P}_{i X Z}=-\left(\eta^{h_{2}} \mathcal{P}_{i} \eta^{h_{2}}\right)\left(\varepsilon \partial_{X} h_{2}\right)+\operatorname{fric}\left(U_{1}, U_{2}\right), i=1,2, \\
-\varepsilon \mu_{i} \mathcal{P}_{i Z X} \partial_{X} h_{2}+\mathcal{P}_{i Z Z}=\left(\eta^{h_{2}} \mathcal{P}_{i} \eta^{h_{2}}\right) \quad i=1,2
\end{gathered}
$$

- $\mathbf{Z}=0$ : On $Z=0$, we have $\eta^{0}=(0,1)$, then from (10) and (11) we obtain

$$
\begin{gathered}
W_{2}=0 \\
\mu_{2} \mathcal{P}_{2 X Z}=-\left(\mathcal{P}_{2 Z Z}-\mathcal{P}_{1 Z Z}\right) \frac{U_{2}^{0}}{\left|U_{2}^{0}\right|} \tan \left(\delta_{0}\right) .
\end{gathered}
$$

\section{Constitutive laws.}

We suppose that $d_{X} \theta=\mathcal{O}(\varepsilon)$. Then from (15) we obtain

$$
\begin{aligned}
& \partial_{Z}\left(\mathcal{P}_{1 Z Z}\right)=-\rho_{1} \cos \theta+\mathcal{O}(\varepsilon), \\
& \partial_{Z}\left(\mathcal{P}_{2 Z Z}\right)=-\rho_{2} \cos \theta+\mathcal{O}(\varepsilon) .
\end{aligned}
$$

If we integrate (24) from $Z>0$ to $S$, we have, up to order $\varepsilon$,

$$
\mathcal{P}_{1 Z Z}=\rho_{1}(S-Z) \cos \theta
$$

therefore, $\mathcal{P}_{1 Z Z}\left(h_{2}\right)=\rho_{1} h_{1} \cos \theta$. Using this last expression, the relations given in (19) and integrating (25) from $Z>0$ to $S$, we have, up to first order

$$
\mathcal{P}_{2 Z Z}^{s}+\mathcal{P}_{2 Z Z}^{f}=\mathcal{P}_{2 Z Z}=\rho_{1} h_{1} \cos \theta+\rho_{2} \cos \theta\left(h_{2}-Z\right) .
$$

The last equation defines the total pressure, $\mathcal{P}_{2 Z Z}$, perpendicular to the base. The constitutive relation for both the grains and the fluid, i. e. $\mathcal{P}_{2 Z Z}^{s}$ and $\mathcal{P}_{2 Z Z}^{f}$, are required to close the model.

The same problem appears if we study a grain-fluid mixture aerial avalanche. See for example [26] and [41]. In order to obtain an expression for the normal stress of both phases, they suppose that both are linear in $Z$. Moreover, they suppose that the component of the stress tensor of the fluid phase normal to the basal surface is proportional to the pressure of a fluid layer, without the solid phase.

We adapt this hypothesis to our case, taking account of the fact that the fluidized layer has an upper layer of fluid. Concretely, we suppose

$$
\mathcal{P}_{2 Z Z}^{f}(Z)=\lambda_{1} \rho_{1} h_{1} \cos \theta+\lambda_{2} \rho_{1} h_{2} \cos \theta\left(h_{2}-Z\right),
$$

where $\lambda_{1}$ and $\lambda_{2}$ are two parameters. Moreover, by (27), we have

$$
\mathcal{P}_{2 Z Z}^{s}(Z)=\rho_{1} h_{1} \cos \theta\left(1-\lambda_{1}\right)+\cos \theta\left(h_{2}-Z\right)\left(\rho_{2}-\lambda_{2} \rho_{1}\right)
$$

The study of the stress transition conditions at a singular surface between two mixtures which do not have the same number of components, is a very difficult subject. Hypothesis (28) can be seen as a fist trial, in the context of this paper. Some earlier papers looking at the problem of interfacial transition conditions with different number of constituents have been included in the references. For example in [25] Hutter et al. study the transition conditions, with application to glaciers where the upper layer is ice and the under layer is a sediment-ice mixture (see also [47], [50], [51]). 
Remark 2 Comparisons with experiments are necessary to define $\lambda_{1}$ and $\lambda_{2}$. Nevertheless we can make some possible choices.

The first simplification is to consider $\lambda_{1}=\lambda_{2}$. In this case the component of the stress tensor of the fluid phase normal to the base is proportional to the pressure of a fluid, without the solid phase in the second layer.

Nevertheless, we prefer at this moment to retain two different parameters. Because the role of $\lambda_{1}$ could be different of that of $\lambda_{2}$. Observe that if we evaluate (28) and (29) for $Z=h_{2}$ we obtain

$$
\mathcal{P}_{2 Z Z}^{f}\left(h_{2}\right)=\lambda_{1} \rho_{1} h_{1} \cos \theta, \quad \mathcal{P}_{2 Z Z}^{s}\left(h_{2}\right)=\rho_{1} h_{1} \cos \theta\left(1-\lambda_{1}\right) ;
$$

Note that $\lambda_{1}$ controls the distribution of the pressure at the interface into the two phases of the second layer.

We have imposed continuity of the component of the stress tensor normal to the base across the interface of the first and second layer (equation (8)), and we observe that it is verified independently of the definition of $\lambda_{1}$. If we want to include an additional condition, for example the continuity of the pressure of the fluid phase of the second layer with the first layer of fluid, then we obtain that $\lambda_{1}=1$. Depending on the material of the second layer, we can also suppose that the fluid that fills the pores of the second layer is nearly isolated of the fluid of the first layer, in this case we can consider $\lambda_{1} \approx 0$.

Independently of the additional hypothesis that we can use to set the distribution of the pressure at the interface between the solid and fluid phase, we have still the parameter $\lambda_{2}$ at our disposal, in order to impose a similar hypothesis to that introduced by Iverson and Delinger, but only for the second layer.

Another possible choise is to fix $\lambda_{1}=\lambda_{2}=\psi_{0}$, where $\psi_{0}$ is the porosity of the second layer. We obtain in this case

$$
\mathcal{P}_{2 Z Z}^{s}=\left(1-\psi_{0}\right)\left(\rho_{1} h_{1}+\rho_{s}\left(h_{2}-Z\right)\right) \cos \theta, \quad \mathcal{P}_{2 Z Z}^{f}=\psi_{0} \rho_{1}\left(h_{1}+h_{2}-Z\right) \cos \theta .
$$

An interesting property of this choice is that $\mathcal{P}_{2 Z Z}$ at height $Z=h 2$ is proportional to the pressure that is obtained in absence of the fluid phase (with proportional constant $\left(1-\psi_{0}\right)$ ). Observe that $\mathcal{P}_{2 Z Z}^{s}$ depends on $\rho_{s}$, the density of the solid phase, and not $\rho_{2}$, the density of the mixture defined by (1). Analogously, $\mathcal{P}_{2 Z Z}^{f}$ is proportional to the pressure (with proportional constant $\psi_{0}$ ) that is obtained in the absence of the solid phase. Moreover, if the porosity of the second layer is zero, then $\mathcal{P}_{2}=\mathcal{P}_{2}^{s}$, which is automatically deduced from this definition.

Finally, the following relations are also considered (see for example [26] and [41]):

$$
\mathcal{P}_{1 X X}=\mathcal{P}_{1 Z Z}, \quad \mathcal{P}_{2 X X}^{s}=K \mathcal{P}_{2 Z Z}^{s}, \quad \mathcal{P}_{2 X X}^{f}=P_{2 Z Z}^{f},
$$

where $K$ measures the anisotropy or normal stress effects in the solid phase. The definition of $K$ can be done in different ways. For example Heinrich et al. in [24] consider $K=1$, other definitions of $K$ can be found in [26]. The effects related to the definition of $K$ in numerical modelling of experimental and natural flows is studied in [41] and [38].

Remark 3 The value $K=1$ corresponds to isotropic conditions, $K \neq 1$ makes 'overburden pressures' different from the normal stresses parallel to the basal surface. In soil mechanics, $K$ corresponds to the earth pressure coefficient, see [41]. For non-Neutonian rheology $K$ may also be different from unity.

Using the previous relations, the following expression for $\mathcal{P}_{2 X X}$ is derived:

$$
\begin{gathered}
\mathcal{P}_{2 X X}=K \mathcal{P}_{2 Z Z}^{s}+\mathcal{P}_{2 Z Z}^{f}= \\
=h_{1} \cos \theta \rho_{1}\left(\lambda_{1}+K\left(1-\lambda_{1}\right)\right)+\left(h_{2}-Z\right) \cos \theta\left(\lambda_{2} \rho_{1}+K\left(\rho_{2}-\lambda_{2} \rho_{1}\right)\right) .
\end{gathered}
$$


Now, replacing (26) and (30) in (14), and using the incompressibility equation (13), we obtain up to second order

$$
\partial_{t}\left(\rho_{1} U_{1}\right)+\rho_{1} \partial_{X} U_{1}^{2}+\rho_{1} \partial_{Z}\left(U_{1} W_{1}\right)+\rho_{1} \partial_{X}(b+S \cos \theta) \varepsilon=-\mu_{1} \partial_{Z}\left(\mathcal{P}_{1 X Z}\right),
$$

and

$$
\begin{gathered}
\partial_{t}\left(\rho_{2} U_{2}\right)+\rho_{2} \partial_{X} U_{2}^{2}+\rho_{2} \partial_{Z}\left(U_{2} W_{2}\right)+\rho_{2} \partial_{X}\left(b+Z \cos \theta+\frac{1}{\rho_{2}}\left[h_{1} \cos \theta \rho_{1}\left(\lambda_{1}+K\left(1-\lambda_{1}\right)\right)+\right.\right. \\
\left.\left.+\left(h_{2}-Z\right) \cos \theta\left(\lambda_{2} \rho_{1}+K\left(\rho_{2}-\lambda_{2} \rho_{1}\right)\right)\right]\right) \varepsilon=-\mu_{2} \partial_{Z}\left(\mathcal{P}_{2 X Z}\right) .
\end{gathered}
$$

\section{Integration process}

In this section, equations (31), (32) and (13) are depth-averaged in the direction normal to the topography. Let us introduce the following notation: we denote by $\bar{U}_{i}, i=1,2$ the velocities of each layer averaged perpendicular to the basal surface:

$$
\bar{U}_{1}=\frac{1}{h_{1}} \int_{h_{2}}^{S} U_{1}(X, Z) d Z, \quad \bar{U}_{2}=\frac{1}{h_{2}} \int_{0}^{h_{2}} U_{2}(X, Z) d Z .
$$

We also denote

$$
\overline{U_{1}^{2}}=\frac{1}{h_{1}} \int_{h_{2}}^{S} U_{1}^{2}(X, Z) d Z, \quad \overline{U_{2}^{2}}=\frac{1}{h_{2}} \int_{0}^{h_{2}} U_{2}^{2}(X, Z) d Z .
$$

Assuming that $d_{X} \theta=\mathcal{O}(\varepsilon)$, then $J=1-Z d_{X} \theta$ is reduced to $J=1$ up to second order. Therefore, (13) reduces to

$$
\begin{aligned}
& \partial_{X}\left(U_{1}\right)+\partial_{Z}\left(W_{1}\right)=0, \\
& \partial_{X}\left(U_{2}\right)+\partial_{Z}\left(W_{2}\right)=0 .
\end{aligned}
$$

I.1) If equation (33) is integrated from $Z=h_{2}$ to $Z=S$, we obtain

$$
0=\partial_{X}\left(h_{1} \bar{U}_{1}\right)-U_{1}(S) \partial_{X} S+W_{1}(S)+U_{1}\left(h_{2}\right) \partial_{X} h_{2}-W_{1}\left(h_{2}\right) .
$$

Now, using the kinematic conditions (16), the following equation is derived:

$$
\partial_{t} h_{1}+\partial_{X}\left(h_{1} \bar{U}_{1}\right)=0 .
$$

I.2) Analogously, by integrating (34) between $Z=0$ and $Z=h_{2}$ we obtain

$$
0=\partial_{X}\left(h_{2} \bar{U}_{2}\right)-U_{2}\left(h_{2}\right) \partial_{X} h_{2}+W_{2}\left(h_{2}\right)-W_{2}(0),
$$

and, using the kinematic condition (16) and the boundary condition (22), the following equation is derived:

$$
\partial_{t} h_{2}+\partial_{X}\left(h_{2} \bar{U}_{2}\right)=0 .
$$

I.3) Let us now proceed with equation (31), integrating it from $Z=h_{2}$ to $Z=S$. We obtain

$$
\begin{gathered}
\rho_{1} \partial_{t}\left(h_{1} \bar{U}_{1}\right)+\rho_{1} \partial_{X}\left(h_{1} \overline{U_{1}^{2}}\right)-\rho_{1} U_{1}(S)\left[\partial_{t}(S)+U_{1}(S) \partial_{X} S-W_{1}(S)\right]+ \\
+\rho_{1} U_{1}\left(h_{2}\right)\left[\partial_{t} h_{2}+U_{1}\left(h_{2}\right) \partial_{X} h_{2}-W_{1}\left(h_{2}\right)\right]+\rho_{1}\left(\int_{h_{2}}^{S}\left(\partial_{X}(b+S \cos \theta)\right) d Z\right) \varepsilon= \\
=-\mu_{1}\left(\mathcal{P}_{1 X Z}(S)-\mathcal{P}_{1 X Z}\left(h_{2}\right)\right) .
\end{gathered}
$$

The expressions of $\mathcal{P}_{1 X Z}(S)$ and $\mathcal{P}_{1 X Z}\left(h_{2}\right)$ are now derived using the boundary conditions and the constitutive laws. 
- Using (17) and (26) and the relation $\mathcal{P}_{1 X X}=\mathcal{P}_{1 Z Z}$ the following expression is obtained:

$$
\mu_{1} \mathcal{P}_{1 Z X}(S)=-\varepsilon \mathcal{P}_{1 X X}(S) \partial_{X} S=-\varepsilon \mathcal{P}_{1 Z Z}(S) \partial_{X} S=0+\mathcal{O}\left(\varepsilon^{2}\right)
$$

- Using (20), we have,

$$
\mu_{1} \mathcal{P}_{1 X Z}\left(h_{2}\right)+\varepsilon \partial_{X} h_{2}\left(\mathcal{P}_{1 Z Z}-\mathcal{P}_{1 X X}\right)=\operatorname{fric}\left(U_{1}, U_{2}\right)+\mathcal{O}\left(\varepsilon^{2}\right) .
$$

Therefore, applying the constitutive law for the fluid layer, that is, $\mathcal{P}_{1 X X}=\mathcal{P}_{1 Z Z}$, the following equality is derived:

$$
\mu_{1} \mathcal{P}_{1 X Z}\left(h_{2}\right)=\operatorname{fric}\left(U_{1}, U_{2}\right)+\mathcal{O}\left(\varepsilon^{2}\right) .
$$

Using the kinematic conditions (16), equation (35) and the expressions obtained for $\mu_{1} \mathcal{P}_{1 X Z}(S)$ (36) and for $\mu_{1} \mathcal{P}_{1 X Z}\left(h_{2}\right)(37)$, we obtain

$$
\rho_{1} \partial_{t}\left(h_{1} \bar{U}_{1}\right)+\rho_{1} \partial_{X}\left(h_{1} \overline{U_{1}^{2}}\right)+\rho_{1}\left(\int_{h_{2}}^{S} \partial_{X}(b+S \cos \theta) d Z\right) \varepsilon=\operatorname{fric}\left(U_{1}, U_{2}\right)+\mathcal{O}\left(\varepsilon^{2}\right)
$$

Now, evaluating the integral, we obtain

$$
\int_{h_{2}}^{S} \partial_{X}(b+S \cos \theta) d Z=h_{1} d_{X} b+\partial_{X}\left(\frac{h_{1}^{2}}{2} \cos \theta\right)+h_{1} \partial_{X}\left(\cos \theta h_{2}\right)-\frac{h_{1}^{2}}{2} \sin \theta d_{X} \theta .
$$

Finally, we obtain the equation

$$
\begin{gathered}
\rho_{1} \partial_{t}\left(h_{1} \bar{U}_{1}\right)+\rho_{1} \partial_{X}\left(h_{1} \overline{U_{1}^{2}}+\varepsilon \frac{h_{1}^{2}}{2} \cos \theta\right)=\varepsilon \rho_{1}\left(-h_{1} d_{X} b+\sin \theta d_{X} \theta \frac{h_{1}^{2}}{2}-\right. \\
\left.-h_{1} \partial_{X}\left(\cos \theta h_{2}\right)\right)+\operatorname{fric}\left(U_{1}, U_{2}\right)+\mathcal{O}\left(\varepsilon^{2}\right) .
\end{gathered}
$$

I.4) Let us now integrate equation (32) from $Z=0$ to $Z=h_{2}$. As in the previous case, we use the kinematic conditions (16) to obtain

$$
\begin{gathered}
\rho_{2} \partial_{t}\left(h_{2} \bar{U}_{2}\right)+\rho_{2} \partial_{X}\left(h_{2} \overline{U_{2}^{2}}\right)+\rho_{2}\left(\int _ { 0 } ^ { h _ { 2 } } \partial _ { X } \left(b+Z \cos \theta+\frac{1}{\rho_{2}}\left[h_{1} \cos \theta \rho_{1}\left(\lambda_{1}+K\left(1-\lambda_{1}\right)\right)+\right.\right.\right. \\
\left.\left.\left.+\left(h_{2}-Z\right) \cos \theta\left(\lambda_{2} \rho_{1}+K\left(\rho_{2}-\lambda_{2} \rho_{1}\right)\right)\right]\right) d Z\right) \varepsilon=-\mu_{2}\left(\mathcal{P}_{2 X Z}\left(h_{2}\right)-\mathcal{P}_{2 X Z}(0)\right) .
\end{gathered}
$$

Let us denote by

$$
r=\frac{\rho_{1}}{\rho_{2}}
$$

where $\rho_{1}$ is the density of the fluid and $\rho_{2}$ is defined by (1).

We obtain

$$
\begin{gathered}
\int_{0}^{h_{2}} \partial_{X}\left(b+Z \cos \theta+\frac{1}{\rho_{2}}\left[h_{1} \cos \theta \rho_{1}\left(\lambda_{1}+K\left(1-\lambda_{1}\right)\right)+\right.\right. \\
\left.\left.+\left(h_{2}-Z\right) \cos \theta\left(\lambda_{2} \rho_{1}+K\left(\rho_{2}-\lambda_{2} \rho_{1}\right)\right)\right]\right) d Z=h_{2} d_{X} b+ \\
\quad+r h_{2}\left(K\left(1-\lambda_{1}\right)+\lambda_{1}\right) \partial_{X}\left(h_{1} \cos \theta\right)+ \\
\quad+\partial_{X}\left(\frac{h_{2}^{2}}{2} \cos \theta\left(r \lambda_{2}+K\left(1-r \lambda_{2}\right)\right)\right)-\frac{h_{2}^{2}}{2} \sin \theta d_{X} \theta
\end{gathered}
$$


Replacing the last expression in (38), and dividing by $\rho_{2}$ and we obtain the equation

$$
\begin{gathered}
\partial_{t}\left(h_{2} \bar{U}_{2}\right)+\partial_{X}\left(h_{2} \overline{U_{2}^{2}}+\varepsilon \frac{h_{2}^{2}}{2} \cos \theta\left(r \lambda_{2}+K\left(1-r \lambda_{2}\right)\right)\right)=-\varepsilon h_{2} d_{X} b- \\
-\varepsilon r h_{2}\left(\lambda_{1}+K\left(1-\lambda_{1}\right)\right) \partial_{X}\left(h_{1} \cos \theta\right)+\varepsilon \frac{h_{2}^{2}}{2} \sin \theta d_{X} \theta-\frac{\mu_{2}}{\rho_{2}}\left(\mathcal{P}_{2 X Z}\left(h_{2}\right)-\mathcal{P}_{2 X Z}(0)\right) .
\end{gathered}
$$

Just as in the previous case, the boundary conditions and the constitutive laws are used to derive $\mu_{2} \mathcal{P}_{2 X Z}\left(h_{2}\right)$ and $\mu_{2} \mathcal{P}_{2 X Z}(0)$.

- Using (20), and $\mathcal{P}_{2 X X}^{s}=K \mathcal{P}_{2 Z Z}^{s}, \mathcal{P}_{2 X X}^{f}=\mathcal{P}_{2 X X}^{f}$ we have

$$
\mu_{2} \mathcal{P}_{2 X Z}\left(h_{2}\right)=\operatorname{fric}\left(U_{1}, U_{2}\right)+\mu_{2} \varepsilon \partial_{X} h_{2} \mathcal{P}_{2 Z Z}^{s}(K-1) .
$$

In [21] Gray introduce the assumption that the Coulomb term is of order $\gamma$ for some $\gamma \in(0,1)$. That is, $\mu_{2}=\tan \left(\delta_{0}\right)=\mathcal{O}\left(\varepsilon^{\gamma}\right)$. Under this assumption, we have

$$
\mu_{2} \mathcal{P}_{2 X Z}\left(h_{2}\right)=\operatorname{fric}\left(U_{1}, U_{2}\right)+\mathcal{O}\left(\varepsilon^{1+\gamma}\right) .
$$

- Using equation (23), we obtain

$$
\mu_{2} \mathcal{P}_{2 X Z}(0)=-\left(\mathcal{P}_{2 Z Z}(0)-\mathcal{P}_{1 Z Z}(0)\right) \frac{U_{2}^{0}}{\left|U_{2}^{0}\right|} \tan \left(\delta_{0}\right) .
$$

Now, using (26) and (27) we have

$$
\left(\mathcal{P}_{2 Z Z}(0)-\mathcal{P}_{1 Z Z}(0)\right)=h_{2} \cos \theta\left(\rho_{2}-\rho_{1}\right)+\mathcal{O}(\varepsilon) .
$$

Therefore, assuming $\tan \left(\delta_{0}\right)=\mathcal{O}\left(\varepsilon^{\gamma}\right)$, we have

$$
\mu_{2} \mathcal{P}_{2 X Z}(0)=-\left(\rho_{2}-\rho_{1}\right) h_{2} \cos \theta \frac{U_{2}^{0}}{\left|U_{2}^{0}\right|} \tan \left(\delta_{0}\right)+\mathcal{O}\left(\varepsilon^{1+\gamma}\right) .
$$

Nevertheless, it is possible to give another expression of the Coulomb term, by including the centripetal acceleration corresponding to the curvature of the bottom (see [26]).

This effect can be easily included and it comes from the derivation of $\mathcal{P}_{2 Z Z}$ in (15), by including a term of order $\varepsilon$. From (15) we obtain up to order $\varepsilon$

$$
\partial_{Z}\left(\mathcal{P}_{2 Z Z}\right)=-\rho_{2} \cos \theta-\rho_{2} U_{2}^{2} d_{X} \theta .
$$

Then, integrating from $Z=0$ to $Z=h_{2}$, and taking into account that $\mathcal{P}_{2 Z Z}\left(h_{2}\right)=$ $\mathcal{P}_{1 Z Z}\left(h_{2}\right)=\rho_{1} h_{1} \cos \theta$, we have

$$
\mathcal{P}_{2 Z Z}(0)=\rho_{1} h_{1} \cos \theta+\rho_{2} h_{2} \cos \theta+\rho_{2} h_{2} \overline{U_{2}^{2}} d X
$$

Finally, the following expression is derived for the Coulomb term

$$
\begin{gathered}
\mu_{2} \mathcal{P}_{2 X Z}(0)=-\left(\mathcal{P}_{2 Z Z}(0)-\mathcal{P}_{1 Z Z}(0)\right) \frac{U_{2}^{0}}{\left|U_{2}^{0}\right|} \tan \left(\delta_{0}\right)= \\
=-\left(\left(\rho_{2}-\rho_{1}\right) h_{2} \cos \theta+\rho_{2} h_{2} \overline{U_{2}^{2}} d_{X} \theta\right) \frac{U_{2}^{0}}{\left|U_{2}^{0}\right|} \tan \left(\delta_{0}\right)+\mathcal{O}\left(\epsilon^{1+\gamma}\right) .
\end{gathered}
$$


Finally, substituting (40) and (41) in (39), we derive the conservation of momentum equation for the second layer

$$
\begin{gathered}
\partial_{t}\left(h_{2} \bar{U}_{2}\right)+\partial_{X}\left(h_{2} \overline{U_{2}^{2}}+\varepsilon \frac{h_{2}^{2}}{2} \cos \theta\left(r \lambda_{2}+K\left(1-r \lambda_{2}\right)\right)\right)= \\
=-\varepsilon h_{2} d_{X} b-\varepsilon r h_{2}\left(\lambda_{1}+K\left(1-\lambda_{1}\right)\right) \partial_{X}\left(h_{1} \cos \theta\right)- \\
-\frac{1}{\rho_{2}} f r i c\left(U_{1}, U_{2}\right)-\left((1-r) h_{2} \cos \theta+h_{2} \bar{U}_{2}^{2} d_{X} \theta\right) \frac{U_{2}^{0}}{\left|U_{2}^{0}\right|} \tan \left(\delta_{0}\right)+\varepsilon \frac{h_{2}^{2}}{2} \sin \theta d_{X} \theta+\mathcal{O}\left(\varepsilon^{1+\gamma}\right) .
\end{gathered}
$$

Remark 4 Another expression of the Coulomb friction term can be obtained including new terms from equation (15). Concretely, in (15) there is another term depending on $\mathcal{P}_{2, Z Z}$. If we include this term we have, up to order $\varepsilon$

$$
\partial_{Z}\left(\mathcal{P}_{2 Z Z}\right)=-\rho_{2} \cos \theta+\left(\varepsilon \mathcal{P}_{2 Z Z}-\rho_{2} U_{2}^{2}\right) d_{X} \theta .
$$

and we also have, up to order $\varepsilon$,

$$
\mathcal{P}_{2 Z Z}\left(h_{2}\right)=\mathcal{P}_{1 Z Z}\left(h_{2}\right)=\rho_{1} h_{1} \cos \theta .
$$

Then, a new expression for $\mathcal{P}_{2} Z Z$, up to order $\varepsilon$, can be obtained integrating the ordinary differential equation (42) with the initial condition (43):

$$
\mathcal{P}_{2 Z Z}(Z)=\left(\int_{Z}^{h_{2}}\left(\rho_{2} \cos \theta-\rho_{2} \partial_{X} \theta U_{i}^{2}(\xi)\right) \exp \left(-\varepsilon d_{X} \theta \xi\right) d \xi\right) \exp \left(\varepsilon d_{X} \theta Z\right)+\rho_{1} h_{1} \cos \theta .
$$

By supposing a constant profile of the velocity we obtain

$$
\mathcal{P}_{2 Z Z}(Z)=\left(\rho_{2} \cos \theta+\rho_{2} d_{X} \theta \bar{U}_{2}^{2}\right) \frac{\exp \left(\left(-h_{2}+Z\right) \varepsilon d_{X} \theta\right)-1}{-\varepsilon d_{X} \theta}+\rho_{1} h_{1} \cos \theta .
$$

Using the Taylor expansion of the exponential we have

$$
\mathcal{P}_{2 Z Z}(0)=\rho_{1} h_{1} \cos \theta+\rho_{2} h_{2} \cos \theta+\rho_{2} h_{2} d_{X} \theta\left(\bar{U}_{2}^{2}-\varepsilon \frac{h_{2} \cos \theta}{2}\right)+\mathcal{O}\left(\left(\varepsilon d_{X} \theta\right)^{2}\right) .
$$

Finally, coming to the original variables the following expression is derived for the Coulomb term

$$
\begin{gathered}
\mu_{2} \mathcal{P}_{2 X Z}(0)=-\left(\mathcal{P}_{2 Z Z}(0)-\mathcal{P}_{1 Z Z}(0)\right) \frac{U_{2}^{0}}{\left|U_{2}^{0}\right|} \tan \left(\delta_{0}\right)= \\
=-\left(g\left(\rho_{2}-\rho_{1}\right) h_{2} \cos \theta+\rho_{2} h_{2} d_{X} \theta\left(\bar{U}_{2}^{2}-\frac{g h_{2} \cos \theta}{2}\right)\right) \frac{U_{2}^{0}}{\left|U_{2}^{0}\right|} \tan \left(\delta_{0}\right) .
\end{gathered}
$$

\section{Final system of equations}

Reverting to the original non-stretched variables, see (12), neglecting the terms of order $\varepsilon^{1+\gamma}$, and by supposing a constant profile of the velocities we obtain the following system:

$$
\left\{\begin{array}{l}
\partial_{t} h_{1}+\partial_{X}\left(h_{1} \bar{U}_{1}\right)=0, \\
\partial_{t}\left(h_{1} \bar{U}_{1}\right)+\partial_{X}\left(h_{1} \bar{U}_{1}^{2}+g \frac{h_{1}^{2}}{2} \cos \theta\right)=-g h_{1} d_{X} b+g \sin \theta d_{X} \theta \frac{h_{1}^{2}}{2}- \\
\quad-g h_{1} \partial_{X}\left(\cos \theta h_{2}\right)+\frac{1}{\rho_{1}} f r i c\left(U_{1}, U_{2}\right), \\
\partial_{t} h_{2}+\partial_{X}\left(h_{2} \bar{U}_{2}\right)=0, \\
\partial_{t}\left(h_{2} \bar{U}_{2}\right)+\partial_{X}\left(h_{2} \bar{U}_{2}^{2}+g \frac{h_{2}^{2}}{2} \cos \theta\left(r \lambda_{2}+K\left(1-r \lambda_{2}\right)\right)\right)=-g h_{2} d_{X} b- \\
\quad-r g h_{2}\left(\lambda_{1}+K\left(1-\lambda_{1}\right)\right) \partial_{X}\left(h_{1} \cos \theta\right)--\frac{1}{\rho_{2}} f r i c\left(U_{1}, U_{2}\right)+g \frac{h_{2}^{2}}{2} \sin \theta d_{X} \theta+\mathcal{T},
\end{array}\right.
$$


where by $\mathcal{T}$, we denote the Coulomb friction term. We observe that this term must be understood as:

$$
\begin{array}{lll}
\text { If }|\mathcal{T}| \geq \sigma_{c} & \Rightarrow & \mathcal{T}=-\left(g(1-r) h_{2} \cos \theta+h_{2} \bar{U}_{2}^{2} d_{X} \theta\right) \frac{\bar{U}_{2}}{\left|\bar{U}_{2}\right|} \tan \left(\delta_{0}\right), \\
\text { If }|\mathcal{T}|<\sigma_{c} & \Rightarrow & \bar{U}_{2}=0,
\end{array}
$$

where $\sigma_{c}=g\left((1-r) h_{2} \cos \theta\right.$. Recall that

$$
r=\frac{\rho_{1}}{\rho_{2}},
$$

where $\rho_{1}$ is the density of the fluid and $\rho_{2}$ is defined in (1).

Remark 5 Heinrich et al. propose in [24] a two layer model to study some type of tsunami produced by submarine avalanches. One of the characteristics of this model is that the equations for the first and second layers are described in different coordinates.

The equations corresponding to the sediment layer are defined in local coordinates over a bottom with constant slope. The equations corresponding to the fluid layer are defined in Cartesian coordinates.

Moreover they consider an uncoupled model, in the sense that the equations for the sediment layer do not depend on the height nor on the velocity of the fluid layer.

Concretely, if $x$ is the Cartesian horizontal coordinate and $X(x)$ the corresponding local coordinate over a non erodible bottom, we denote by $\mathcal{H}_{1}(x)$ and $\mathcal{H}_{2}(x)$ the heights of the fluid and the sediment layer in the vertical direction $\left.\mathcal{H}_{1}(x)=h_{1}(X(x)) \cos \theta(X(x)), \mathcal{H}_{2}(x)=h_{2}(X(x)) \cos \theta(X(x))\right)$, and if the horizontal velocity for the fluid layer is denoted by $u_{1}$, the model proposed by Heinrich et al. can be written as

$$
\left\{\begin{array}{l}
\partial_{t} \mathcal{H}_{1}+\partial_{x}\left(\mathcal{H}_{1} u_{1}\right)=0 \\
\partial_{t}\left(\mathcal{H}_{1} u_{1}\right)+\partial_{x}\left(\mathcal{H}_{1} \bar{u}_{1}^{2}+g \frac{\mathcal{H}_{1}^{2}}{2}\right)=-g \mathcal{H}_{1} d_{x}\left(b+\mathcal{H}_{2}\right) \\
\partial_{t} h_{2}+\partial_{X}\left(h_{2} \bar{U}_{2}\right)=0 \\
\partial_{t}\left(h_{2} \bar{U}_{2}\right)+\partial_{X}\left(h_{2} \bar{U}_{2}^{2}+g(1-r) \frac{h_{2}^{2}}{2} \cos \theta\right)=-g(1-r) h_{2} d_{X} b+\mathcal{T}
\end{array}\right.
$$

They proposed to solve this system in the following way: Firstly the height of the sediment layer is computed in local coordinates by using the third and fourth equations of (48). Then, the bottom, obtained as the sum of the fixed bottom plus the sediment layer, is recalculated in Cartesian coordinates. And finally, the evolution of the fluid layer is computed by using the first and second equations of (48).

Therefore, the main difference with the model proposed here is that, in our case, the complete model is described in local coordinates and the two layers are fully coupled by the pressure terms.

It is easy to see, that the model proposed by Heinrich et al. could be obtained from the one presented here by assuming the rigid lid hypothesis in the derivation of the equations for the sediment layer. Indeed, rewriting the term

$$
-r g h_{2}\left(\lambda_{1}+K\left(1-\lambda_{1}\right)\right) \partial_{X}\left(h_{1} \cos \theta\right)
$$

in the last equation (45), under the rigid lid assumption

$$
b+\left(h_{1}+h_{2}\right) \cos \theta=c s t
$$

the following equation for the momentum conservation of the second layer is obtained

$$
\begin{aligned}
\partial_{t}\left(h_{2} \bar{U}_{2}\right)+ & \partial_{X}\left(h_{2} \bar{U}_{2}^{2}+g \frac{h_{2}^{2}}{2} \cos \theta\left(\lambda_{2} r+K\left(1-\lambda_{2} r\right)-r\left(\lambda_{1}+K\left(1-\lambda_{1}\right)\right)\right)\right)= \\
& -g h_{2}\left(1-r\left(\lambda_{1}+K\left(1-\lambda_{1}\right)\right)\right) d_{X} b-\frac{1}{\rho_{2}} f r i c\left(U_{1}, U_{2}\right)+
\end{aligned}
$$




$$
+g \frac{h_{2}^{2}}{2} \sin \theta d_{X} \theta\left(1-r\left(\lambda_{1}+K\left(1-\lambda_{1}\right)\right)\right)+\mathcal{T},
$$

where actually the coupled term disappears. Compare (49) with the last equation of (48).

\section{Properties of the model}

In this section we study the properties of the proposed model. We fix the friction term between the layers $\operatorname{fric}\left(U_{1}, U_{2}\right)$ to

$$
\operatorname{fric}\left(U_{1}, U_{2}\right)=-\mathcal{K}_{i n} \cdot\left(\bar{U}_{1}-\bar{U}_{2}\right), \quad \text { with } \mathcal{K}_{i n}=\rho_{1} K_{i n}\left|\bar{U}_{1}-\bar{U}_{2}\right|,
$$

being $K_{\text {in }}$ a positive constant. In this case, the model reduces to

$$
\left\{\begin{array}{c}
\partial_{t} h_{1}+\partial_{X}\left(h_{1} \bar{U}_{1}\right)=0 \\
\partial_{t}\left(h_{1} U_{1}\right)+\partial_{X}\left(h_{1} \bar{U}_{1}^{2}+g \cos \theta \frac{h_{1}^{2}}{2}\right)= \\
=-g h_{1} d_{X} b+g \sin \theta d_{X} \theta \frac{h_{1}^{2}}{2}-g h_{1} \partial_{X}\left(\cos \theta h_{2}\right)-K_{i n}\left|\bar{U}_{1}-\bar{U}_{2}\right|\left(\bar{U}_{1}-\bar{U}_{2}\right) \\
\partial_{t} h_{2}+\partial_{X}\left(h_{2} \bar{U}_{2}\right)=0 \\
\partial_{t}\left(h_{2} \bar{U}_{2}\right)+\partial_{X}\left(h_{2} \bar{U}_{2}^{2}+g \cos \theta \frac{h_{2}^{2}}{2} \Lambda_{2}\right)=-g h_{2} d_{X} b-r g h_{2} \Lambda_{1} \partial_{X}\left(h_{1} \cos \theta\right)- \\
\quad-r K_{i n}\left|\bar{U}_{1}-\bar{U}_{2}\right|\left(\bar{U}_{2}-\bar{U}_{1}\right)+g \frac{h_{2}^{2}}{2} \sin \theta d_{X} \theta+\mathcal{T}
\end{array}\right.
$$

where we denoted

$$
\Lambda_{1}=\lambda_{1}+K\left(1-\lambda_{1}\right), \quad \Lambda_{2}=r \lambda_{2}+K\left(1-r \lambda_{2}\right) .
$$

Remark 6 Observe that for $K=1$ we obtain $\Lambda_{1}=1$ and $\Lambda_{2}=1$. Then, in this case the system is independent of the parameters $\lambda_{1}$ and $\lambda_{2}$.

The coefficient $K$ is defined according to the motion of the second layer (see [41]):

$$
K= \begin{cases}K_{a c t} & \text { if } \partial_{X} \bar{U}_{2}>0 \\ K_{\text {pas }} & \text { if } \partial_{X} \bar{U}_{2}<0\end{cases}
$$

with

$$
K_{\text {act } / \text { pas }}=2 \sec ^{2} \phi\left(1 \mp\left(1-\cos ^{2} \phi \sec ^{2} \delta_{0}\right)^{1 / 2}\right)-1,
$$

being $\phi$ the internal friction angle.

The following result can be proved

TheOREM 1 System (50) has the following properties

i) it admits an entropy dissipation inequality,

$$
\begin{gathered}
\partial_{t}\left(\frac{r \Lambda_{1} h_{1} \bar{U}_{1}^{2}+h_{2} \bar{U}_{2}^{2}}{2}+g b\left(r \Lambda_{1} h_{1}+h_{2}\right)+g \cos \theta \frac{r \Lambda_{1} h_{1}^{2}+\Lambda_{2} h_{2}^{2}}{2}+g \cos \theta r \Lambda_{1} h_{1} h_{2}\right)+ \\
+\partial_{X}\left(r \Lambda_{1} h_{1} \bar{U}_{1}\left(\frac{\bar{U}_{1}^{2}}{2}+g b+\cos \theta\left(h_{1}+h_{2}\right)\right)+h_{2} \bar{U}_{2}\left(\frac{\bar{U}_{2}^{2}}{2}+g b+g \cos \theta\left(r \Lambda h_{1}+\Lambda_{2} h_{2}\right)\right)\right) \leq \\
\leq-r K_{i n}\left|\bar{U}_{1}-\bar{U}_{2}\right|\left(U_{2}-U_{1}\right)\left(U_{2}-\Lambda_{1} U_{1}\right)-g\left((1-r) h_{2} \cos \theta+h_{2} d_{X} \theta\left(\bar{U}_{2}^{2}-\frac{g h_{2} \cos \theta}{2}\right)\right)\left|\bar{U}_{2}\right| \tan \left(\delta_{0}\right)+ \\
+g \frac{h_{2}^{2}}{2} U_{2}\left(1-\Lambda_{2}\right) \sin \theta \partial_{X} \theta .
\end{gathered}
$$


ii) it preserves the steady state corresponding to water at rest over a stationary sediment, that is a stationary solution verifying:

$$
\begin{gathered}
\bar{U}_{1}=0, \quad \bar{U}_{2}=0, \\
b+\left(h_{1}+h_{2}\right) \cos \theta=c s t, \\
\left|\left(\Lambda_{2}-r \Lambda_{1}\right) \partial_{x}\left(b+h_{2} \cos \theta\right)+\left(1-\Lambda_{2}\right)\left(\partial_{x} b-\frac{h_{2}}{2} \sin \theta \partial_{x} \theta\right)\right| \leq(1-r) \tan \left(\delta_{0}\right),
\end{gathered}
$$

iii) for constant slope $d_{X} \theta=0$ and $K=1$, the system (50) gives an exact solution to the free surface Euler system with hydrostatic assumption.

\section{PROOF}

To prove $i$ ) we first obtain an equation for $\bar{U}_{1}$ by combining the first and second of equations (50):

$$
\partial_{t}\left(\bar{U}_{1}\right)+\partial_{X}\left(\frac{\bar{U}_{1}^{2}}{2}+g b+g \cos \theta\left(h_{1}+h_{2}\right)\right)=-\frac{K_{i n}}{h_{1}}\left|\bar{U}_{1}-\bar{U}_{2}\right|\left(\bar{U}_{1}-\bar{U}_{2}\right) .
$$

Also using the third and fourth of equations (50), we obtain

$$
\begin{gathered}
\partial_{t}\left(\bar{U}_{2}\right)+\partial_{X}\left(\frac{\bar{U}_{2}}{2}+g \cos \theta\left(r \Lambda_{1} h_{1}+\Lambda_{2} h_{2}\right)+g b\right)=-g\left(\cos \theta(1-r)+\partial_{X} \theta\left(\bar{U}_{2}^{2}-\frac{g h_{2} \cos \theta}{2}\right)\right) \frac{\bar{U}_{2}}{\left|\bar{U}_{2}\right|} \tan \left(\delta_{0}\right)- \\
-r \frac{K_{\text {in }}}{h_{2}}\left|\bar{U}_{1}-\bar{U}_{2}\right|\left(\bar{U}_{2}-\bar{U}_{1}\right)-g \frac{h_{2}}{2}\left(1-\Lambda_{2}\right) \partial_{X} \cos \theta
\end{gathered}
$$

Now, by multiplying the equation on (50) by $\frac{\bar{U}_{1}^{2}}{2}+g b+g \cos \theta\left(h_{1}+h_{2}\right)$ and (55) by $h_{1} \bar{U}_{1}$ we obtain

$$
\begin{gathered}
\partial_{t}\left(\frac{h_{1} \bar{U}_{1}^{2}}{2}+g b h_{1}+g \cos \theta \frac{h_{1}^{2}}{2}\right)+\partial_{X}\left(h_{1} \bar{U}_{1}\left(\frac{\bar{U}_{1}^{2}}{2}+g b+g \cos \theta\left(h_{1}+h_{2}\right)\right)\right)= \\
=-K_{i n}\left|\bar{U}_{1}-\bar{U}_{2}\right| \bar{U}_{1}\left(\bar{U}_{1}-\bar{U}_{2}\right)-g \cos \theta h_{2} \partial_{t} h_{1} .
\end{gathered}
$$

Analogously, multiplying the third equation on (50) by $\frac{\overline{U_{2}}}{2}+g \cos \theta\left(r \Lambda_{1} h_{1}+\Lambda_{2} h_{2}\right)+g b$ and (56) by $h_{2} \bar{U}_{2}$ we obtain

$$
\begin{gathered}
\partial_{t}\left(\frac{h_{2} \bar{U}_{2}^{2}}{2}+g b h_{2}+g \cos \theta \Lambda_{2} \frac{h_{2}^{2}}{2}\right)+\partial_{X}\left(h_{2} \bar{U}_{2}\left(\frac{\bar{U}_{2}}{2}+g \cos \theta\left(r \Lambda_{1} h_{1}+\Lambda_{2} h_{2}\right)+g b\right)\right)= \\
=-g \cos \theta r \Lambda_{1} h_{1} \partial_{t}\left(h_{2}\right)-r K_{i n}\left|\bar{U}_{1}-\bar{U}_{2}\right| \bar{U}_{2}\left(\bar{U}_{2}-\bar{U}_{1}\right)- \\
-g\left((1-r) h_{2} \cos \theta+h_{2} \partial_{X} \theta\left(\bar{U}_{2}^{2}-\frac{g h_{2} \cos \theta}{2}\right)\right)\left|\bar{U}_{2}\right| \tan \left(\delta_{0}\right)-g \frac{h_{2}^{2}}{2}\left(1-\Lambda_{2}\right) U_{2} \partial_{X} \cos \theta .
\end{gathered}
$$

Finally, if equation (57) is multiplied by $r \Lambda_{1}$ and added to (58), we obtain (51).

To proof $i i)$, it is easy to verify that the three first equations are trivially satisfied. To verify the last equation, taking into account (53), we deduce

$$
\mathcal{T}=g h_{2} \cos \theta\left(\left(\Lambda_{2}-r \Lambda_{1}\right) \partial_{x}\left(b+h_{2} \cos \theta\right)+\left(1-\Lambda_{2}\right)\left(\partial_{x} b-\frac{h_{2}}{2} \sin \theta \partial_{x} \theta\right)\right)
$$

and applying (54), we have

$$
|\mathcal{T}| \leq g h_{2}(1-r) \cos \theta \tan \left(\delta_{0}\right)=\sigma_{c}
$$

and then $\bar{U}_{2}=0$.

The proof of $i v)$ is easy, taking into account that $P=\left(h_{1}(t, X)+h_{2}(t, X)-Z\right) g \cos \theta$. 
Remark 7 The inequality (51) is just the energy conservation for smooth solutions, supposing $K_{\text {in }}=0, \delta_{0}=0$ and $\left(1-\Lambda_{2}\right) \partial_{x} \theta=0$ (that is, $\Lambda_{2}=1$ or $\theta$ constant).

Remark 8 Observe that the inequality (54) is independent of $\lambda_{1}, \lambda_{2}$ and $\partial_{x} \theta$ when $K=1$. For $K=1$ (54) reduces to

$$
\left|\partial_{x}\left(b+h_{2} \cos \theta\right)\right| \leq \tan \left(\delta_{0}\right) .
$$

As the equation of the interface between the fluid and the sediment material is defined by $b+h_{2} \cos \theta$, the previous condition implies that the slope of the interface is smaller than $\delta_{0}$.

In the case $K \neq 1$, (54) relates the values of $K$ to the curvature of the bottom, the parameters $\lambda_{1}, \lambda_{2}$ and the ratio between the densities of the fluid and the granular material, $r$ (see 2).

Observe that for stationary solutions verifying $U_{2}=0$ then $\partial_{X} U_{2}=0$. Then, if we consider for this case $K=\left(K_{\text {act }}+K_{\text {pas }}\right) / 2$, we have

$$
K=1+\tan ^{2} \phi
$$

By another way, we can study the profiles verifying the equality in (54). We consider a domain $[0, L]$ and we impose the value of the interface at $x=L$, then for $\delta_{0}, K, \lambda_{1}, \lambda_{2}$ and $r$ fixed we have the equation

$$
\left\{\begin{array}{l}
\alpha \cos \theta \partial_{x} h_{2}+\left(\left(\alpha+\frac{\beta}{2}\right) \partial_{x} \cos \theta\right) h_{2}=(1-r) \tan \left(\delta_{0}\right)-(\alpha+\beta) \partial_{x} b \\
\left.\left(h_{2} \cos \theta+b\right)\right|_{x=L}=A
\end{array}\right.
$$

with $\alpha=\Lambda_{2}-r \Lambda_{1}, \beta=1-\Lambda_{2}$. The solution is

$$
h_{2}(x)=\left(I(x)-I(L)+\cos \theta^{\beta /(2 \alpha)}(A-b(L))\right) \cos \theta^{-1+\beta /(2 \alpha)}
$$

where

$$
I(x)=\int_{0}^{x}\left(\frac{(1-r) \tan \left(\delta_{0}\right)}{\alpha} \cos \theta^{\beta /(2 \alpha)}-\left(1+\frac{\beta}{\alpha}\right) \sin \theta \cos \theta^{-1-\beta /(2 \alpha)}\right) d x .
$$

In Figure 2 we present two examples of the profiles that we obtain for two different bottom topographies for different values of $K$. From $K=1$ to $K=2$ they correspond to the definition (60) with $\phi$ from 0 to 45 degrees. In both examples we have set $\lambda_{1}=\lambda_{2}=\psi_{0}, \psi_{0}=0.2, \delta_{0}=28$ degrees and $r=0.2$.

Figure 2(a) corresponds to a bottom with constant slope equal to 15 degrees, where $L=2$ meters and the interface at $x=L$ is $A=2$. In this example we observe that the interfaces obtained for $K>1$ are over the interface corresponding to $K=1$.

The bottom of Figure $2(b)$ is defined by $b(x)=-\ln (\cos (x))$, moreover $L=1.5$ and $A=3.5$. In this example we observe that by the influence of the curvature of the bottom the interfaces corresoponding to $K>1$ are under the interface obtained for $K=1$.

\section{Numerical scheme: rewriting the model}

In this section we describe the numerical scheme that we propose to discretize model (45). We propose a well-balanced finite volume method that exactly preserves the solutions corresponding to water at rest and no movement of the sediment layer verifying (52), (53), (54); and up to second order all stationary solutions. In Subsection 4.1 we introduce the numerical scheme, and we study its properties.

However, before defining the numerical scheme we begin by rewriting the proposed model in Cartesian coordinates.

We remark that model (45) is written in local coordinates over a non-erodible bottom. In order to solve the problem of defining a proper mesh for an arbitrary topography, we propose to rewrite 


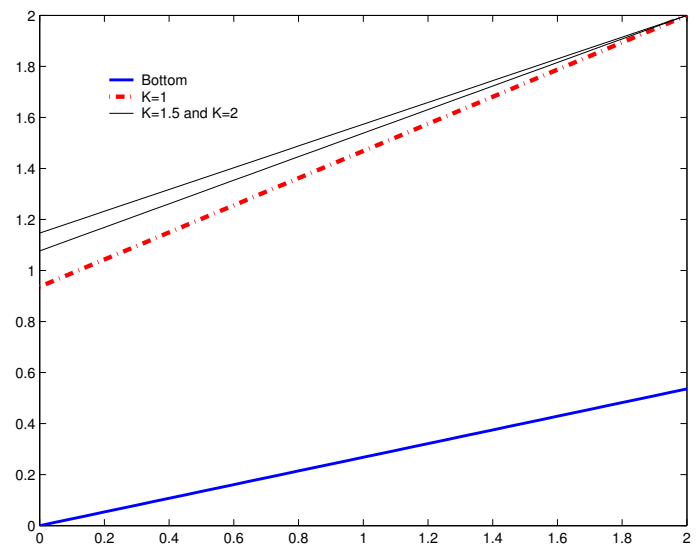

(a) Bottom with constant slope

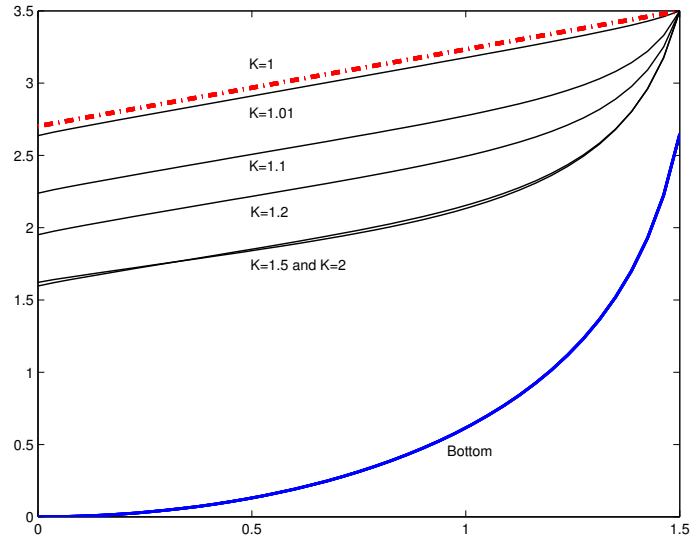

(b) Bottom with curvature

Figure 2: Stationary interface profiles depending on the values of $K$

the model (45) in Cartesian coordinates. To do this, the following rule is used: for a given function $f(X(x))$,

$$
\text { as } \quad \partial x=\cos \theta \partial X \quad \Rightarrow \quad \frac{\partial f}{\partial X}=\cos \theta \frac{\partial f}{\partial x} .
$$

Introducing the notation

$$
H_{i}=\frac{h_{i}}{\cos \theta}, \quad Q_{i}=H_{i} \bar{U}_{i}, \quad i=1,2,
$$

equations (45) can be written as

$$
\left\{\begin{array}{l}
\partial_{t} H_{1}+\partial_{x}\left(Q_{1} \cos \theta\right)=0 \\
\partial_{t}\left(Q_{1}\right)+\partial_{x}\left(H_{1} \bar{U}_{1}^{2} \cos \theta+g \frac{H_{1}^{2}}{2} \cos ^{3} \theta\right)=-g H_{1} \cos \theta d_{x} b+ \\
\quad+g \frac{H_{1}^{2}}{2} \sin \theta \cos ^{2} \theta d_{x} \theta-g H_{1} \cos \theta \partial_{x}\left(H_{2} \cos ^{2} \theta\right)+\frac{f r i c\left(U_{1}, U_{2}\right)}{\cos \theta \rho_{1}}, \\
\partial_{t} H_{2}+\partial_{x}\left(Q_{2} \cos \theta\right)=0 \\
\partial_{t}\left(Q_{2}\right)+\partial_{x}\left(H_{2} \bar{U}_{2}^{2} \cos \theta+g \Lambda_{2} \frac{H_{2}^{2}}{2} \cos ^{3} \theta\right)=-g H_{2} \cos \theta d_{x} b+ \\
\quad+g \frac{H_{2}^{2}}{2} \sin \theta \cos ^{2} \theta \partial_{x} \theta-r \Lambda_{1} g H_{2} \cos \theta \partial_{x}\left(H_{1} \cos ^{2} \theta\right)-\frac{f r i c\left(U_{1}, U_{2}\right)}{\cos \theta \rho_{2}}+\frac{\mathcal{T}}{\cos \theta},
\end{array}\right.
$$

where $\mathcal{T}$ is defined by

$$
\begin{aligned}
& \text { If }|\mathcal{T}| \geq \sigma_{c} \quad \Rightarrow \mathcal{T}=-\left(g(1-r) H_{2} \cos ^{2} \theta+H_{2} \cos \theta \bar{U}_{2}^{2} d_{x}(\sin \theta)\right) \frac{Q_{2}}{\left|Q_{2}\right|} \tan \delta_{0}, \\
& \text { If }|\mathcal{T}|<\sigma_{c} \Rightarrow Q_{2}=0,
\end{aligned}
$$

where $\sigma_{c}=g(1-r) H_{2} \cos ^{2} \theta \tan \left(\delta_{0}\right)$.

\subsection{Well-balanced finite volume method}

In this subsection we present the finite volume method that we use to discretize system (62). There are several difficulties related to the discretization of this system: As we describe below, we can rewrite (62) under the structure of a hyperbolic system with a conservative term, a non-conservative product and two types of source terms (see equation (65) below), where 
i) the flux function does not only depend on the vector of unknowns, but also on $\theta(x)$;

ii) the coupling term is a non-conservative product $B(W) \partial_{x} W$. In general, it is not well defined nor as a distribution and the choice of a family of paths is necessary (see [14]);

iii) the source terms $G_{1}$ and $G_{2}$, are defined as functions of the fixed topography. Their numerical discretization can be treated by following the ideas given in [12] or [13] in the framework of a system of balance laws or by rewriting the system for an extended variable and an extra equation in such a way that the source terms are written in the form of non-conservative products (see [36]);

iv) the source term corresponding to the Coulomb term presents a different difficulty. We propose a two step method combining a well-balanced discretization of the Coulomb term and the numerical treatment introduced by Mangeney et al. in [31]. The numerical method constructed in this way is exactly well balanced for the solutions corresponding to water at rest and no movement of the sediment layer given by (52), (53) and (54).

We can rewrite model (62) under the form of a hyperbolic system with a conservative product, a non-conservative term and source terms:

$$
\partial_{t} W+\partial_{x} F(\theta, W)=G_{1}(x, W)+G_{2}(x, W)+B(W) \partial_{x} W+T,
$$

where

$$
\begin{aligned}
& W=\left(\begin{array}{c}
H_{1} \\
Q_{1} \\
H_{2} \\
Q_{2}
\end{array}\right), \quad F(\theta, W)=\left(\begin{array}{c}
Q_{1} \cos \theta \\
\frac{Q_{1}^{2}}{H_{1}} \cos \theta+g \frac{H_{1}^{2}}{2} \cos ^{3} \theta \\
\frac{Q_{2}^{2}}{H_{2}} \cos \theta+g \Lambda_{2} \frac{H_{2}^{2}}{2} \cos ^{3} \theta
\end{array}\right), \\
& G_{1}=\left(\begin{array}{c}
0 \\
-g H_{1} \cos \theta d_{x} b \\
0 \\
-g H_{2} \cos \theta d_{x} b
\end{array}\right), \quad G_{2}=\left(\begin{array}{c}
0 \\
-g \frac{H_{1}}{2}\left(\frac{H_{1}}{2}+2 H_{2}\right) \cos \theta \partial_{x}\left(\cos ^{2} \theta\right) \\
0 \\
-g \frac{H_{2}}{2}\left(\frac{H_{2}}{2}+2 r \Lambda_{1} H_{1}\right) \cos \theta \partial_{x}\left(\cos ^{2} \theta\right)
\end{array}\right), \\
& B(W)=\left(\begin{array}{cccc}
0 & 0 & 0 & 0 \\
0 & 0 & -g H_{1} \cos ^{3} \theta & 0 \\
0 & 0 & 0 & 0 \\
-r \Lambda_{1} g H_{2} \cos ^{3} \theta & 0 & 0 & 0
\end{array}\right), \quad T=\left(\begin{array}{c}
0 \\
0 \\
0 \\
\mathcal{T} / \cos \theta
\end{array}\right) .
\end{aligned}
$$

Note that the terms $\partial_{x}\left(H_{2} \cos ^{2} \theta\right)$ and $\partial_{x}\left(H_{1} \cos ^{2} \theta\right)$ of the second and fourth equations on (62) contributes to (65) in the definition of the non-conservative term $B(W) \partial_{x} W$ and in the definition of $G_{2}$.

Remark 9 Observe also that $G_{2}$ can be written in terms of $\partial_{x}\left(\cos ^{3} \theta\right)$, nevertheless we propose to define $G_{2}$ in terms of $\cos \theta \partial_{x}\left(\cos ^{2} \theta\right)$, motivated by the discretization that we proposed. The purpose is that we want to obtain an exactly well-balanced numerical scheme for water at rest:

$$
\bar{U}_{1}=\bar{U}_{2}=0, \quad b+H_{2} \cos ^{2} \theta=c s t, \quad H_{1} \cos ^{2} \theta=c s t,
$$

that is defined in terms of $\cos ^{2} \theta$.

Remark 10 System (65) can be written in nonconservative form,

$$
\partial_{t} W+\mathcal{A}(\theta, W) \partial_{x} W=S(\theta, W)
$$


where $S(\theta, W)=G_{1}+G_{2}-\partial_{\theta} F+T$. And where $\mathcal{A}(\theta, W)$ defines the transport matrix of the system:

$$
\mathcal{A}(\theta, W)=\left(\begin{array}{cccc}
0 & \cos \theta & 0 & 0 \\
-U_{1}^{2} \cos \theta+g H_{1} \cos ^{3} \theta & 2 U_{1} \cos \theta & g H_{1} \cos ^{3} \theta & 0 \\
0 & 0 & 0 & \cos \theta \\
r \Lambda_{1} g H_{2} \cos ^{3} \theta & 0 & -U_{2}^{2} \cos \theta+\Lambda_{2} g H_{2} \cos ^{3} \theta & 2 U_{2} \cos \theta
\end{array}\right),
$$

where $U_{i}=Q_{i} / H_{i}$ represents the averaged velocity of the $i$-th layer, and $r=\frac{\rho_{1}}{\rho_{2}}$.

This matrix is similar to the one obtained in the well-known two-layer Shallow Water system (see [36] for example). Unfortunally no explicit expressions of the eigenvectors of the system can be obtained. The characeristic equation of the system is:

$$
\left(\lambda^{2}-2 U_{1} \lambda+U_{1}^{2}-g H_{1} \cos ^{2} \theta\right)\left(\lambda^{2}-2 U_{2} \lambda+U_{2}^{2}-g \Lambda_{2} H_{2} \cos ^{2} \theta\right)=r \Lambda_{1} g^{2} H_{1} H_{2} \cos ^{4} \theta .
$$

It is not easy to verify the genuinely nonlinear character of the 4 characteristic fields, as the eigenvalues and eigenvectors can not be written explicitely in a simple manner. Nevertheless, this fact is easily proved in the case $r=0$ as, in this case, the system reduces to a decoupled system of Shallow Water and Savage-Hutter equations. In this case the eigenvalues are those corresponding to each layer separately. Then, a continuity argument ensures the genuinely nonlinear character of the 4 characteristic fields when $r$ is close to zero.

In the case $r \approx 1$, in [46] authors gives an approximation of the eiganvalues for the two-layer Shallow Water equations. The case $r \approx 1$ is the situation arising for two fluid with different densities in many oceanographical flows. In the context of submarine avalanches we suppose two different materials, then we are closer to the case $r \approx 0$.

Although, following [46], we can also give a approximation of the eiganvalues for $r \approx 1$. We obtain:

$$
\begin{gathered}
\lambda_{\text {ext }}^{ \pm} \approx \frac{U_{1} H_{1}+U_{2} H_{2}}{H_{1}+\Lambda_{2} H_{2}} \pm\left(g\left(H_{1}+\Lambda_{2} H_{2}\right)\right)^{\frac{1}{2}} \cos \theta \\
\lambda_{\text {int }}^{ \pm} \approx \frac{U_{1} \Lambda_{2} H_{2}+U_{2} H_{1}}{H_{1}+\Lambda_{2} H_{2}} \pm\left(g\left(1-r \frac{\Lambda_{1}}{\Lambda_{2}}\right) \frac{H_{1} H_{2} \Lambda_{2} \cos ^{2} \theta}{\left(H_{1}+\Lambda_{2} H_{2}\right)}\left[1-\frac{\left(U_{1}-U_{2}\right)^{2}}{g\left(1-r \frac{\Lambda_{1}}{\Lambda_{2}}\right)\left(H_{1}+\Lambda_{2} H_{2}\right) \cos ^{2} \theta}\right]\right)^{\frac{1}{2}} .
\end{gathered}
$$

From equation (69) we can observe that the internal eigenvalues may become complex. This situation occurs when they verify, approximately, the following inequality:

$$
\frac{\left(U_{1}-U_{2}\right)^{2}}{g\left(1-r \frac{\Lambda_{1}}{\Lambda_{2}}\right)\left(H_{1}+\Lambda_{2} H_{2}\right) \cos ^{2} \theta}>1 .
$$

In this case, the system loses its hyperbolic character. These situations are related with the appearance of shear instabilities that may lead, in real flows, to intense mixing of the two layers. While, in practice, this mixture partially dissipates the energy, in numerical experiments these interface disturbances grow and overwhelm the solution. Clearly, we cannot expect to simulate these phenomena with a two-immiscible-layer model. Therefore, the inequality (70) in fact gives the range of validity of a model based on the equations (65). In this work only the case where the matrix $\mathcal{A}(\theta, W)$ has real eigenvalues is considered, i.e. the system is supposed to be strictly hyperbolic.

As the source terms modeling the friction between the two layers are discretized semi-implicitly, they do not appear in the finite volume discretization, therefore, they are supposed to be zero in this section.

For the discretization of the system, computing cells $I_{i}=\left[x_{i-1 / 2}, x_{i+1 / 2}\right]$ are considered. For simplicity, we suppose that these cells have constant size $\Delta x$. Let us define $x_{i+\frac{1}{2}}=i \Delta x$ and by $x_{i}=(i-1 / 2) \Delta x$, the center of the cell $I_{i}$. Let $\Delta t$ be the constant time step and define $t^{n}=n \Delta t$. 
We denote by $W_{i}^{n}$ the approximation of the cell averages of the exact solution provided by the numerical scheme:

$$
W_{i}^{n} \cong \frac{1}{\Delta x} \int_{x_{i-1 / 2}}^{x_{i+1 / 2}} W\left(x, t^{n}\right) d x .
$$

The source terms $G_{1}$ and $G_{2}$ are discretized following the ideas introduced in [13] and [36]. The discretization of $B(W) \partial_{x} W$ firstly requires to interpret this term as a Borel measure (see [14]), depending on the choice of a family of paths linking given states. Here the family of segments are considered as in [36].

The dependence of the flux function on $\theta(x)$, makes it difficult to obtain the desired exact well-balanced property for water at rest (see [9]). Following the same ideas that have been exposed in Remark 9, we propose to consider the flux function $F(\theta, W)$ as a function of $\cos \theta$ and $\cos ^{2} \theta$. More precisely,

$$
F(\theta, W)=\mathcal{F}\left(\cos \theta, \cos ^{2} \theta, W\right), \text { with } \mathcal{F}(\alpha, \beta, W)=\left(\begin{array}{c}
Q_{1} \alpha \\
\frac{Q_{1}^{2}}{H_{1}} \alpha+g \frac{H_{1}^{2}}{2} \alpha \beta \\
Q_{2} \alpha \\
\frac{Q_{2}^{2}}{H_{2}} \alpha+g \Lambda_{2} \frac{H_{2}^{2}}{2} \alpha \beta
\end{array}\right) .
$$

Finally, as mentioned before, the discretization of the source term $T(W)$ corresponding to the Coulomb friction term is critical to simulate properly the landslides and to preserve the stationary solutions corresponding to water at rest and no movement of the sediment layer verifying (52), (53) and (54). We propose a two-step numerical scheme to treat the Coulomb friction term.

Let us suppose that the values $W_{i}^{n}$ are known. In order to advance in time we proceed as follows:

- First Step. We define $W_{i}^{*}=\left[\begin{array}{llll}H_{1, i}^{*} & Q_{1, i}^{*} & H_{2, i}^{*} & Q_{2, i}^{*}\end{array}\right]^{T}$ as

$$
W_{i}^{*}=W_{i}^{n}-\frac{\Delta t}{\Delta x}\left(\mathcal{D} \mathcal{F}_{i-1 / 2}^{n,+}+\mathcal{D} \mathcal{F}_{i+1 / 2}^{n,-}\right),
$$

where $\mathcal{D F}_{i+1 / 2}^{n, \pm}=\mathcal{D} \mathcal{F}_{i+1 / 2}^{ \pm}\left(W_{i}^{n}, W_{i+1}^{n}\right)$ are the generalized Roe flux difference computed using the family of segments.

$$
\begin{aligned}
\mathcal{D F}_{i+1 / 2}^{ \pm}\left(W_{i}, W_{i+1}\right)= & \frac{1}{2}\left\{\left(\mathcal{F}\left(\cos \theta_{i+1 / 2},\left(\cos ^{2} \theta\right)_{i+1 / 2}, W_{i+1}\right)\right.\right. \\
- & \mathcal{F}\left(\cos \theta_{i+1 / 2},\left(\cos ^{2} \theta\right)_{i+1 / 2}, W_{i}\right)+S_{3, i+1 / 2}\left(\cos ^{2} \theta_{i+1}-\cos ^{2} \theta_{i}\right) \\
+ & S_{4, i+1 / 2}\left(\cos \theta_{i+1}-\cos \theta_{i}\right)-S_{1, i+1 / 2}\left(b_{i+1}-b_{i}\right) \\
- & \left.S_{2, i+1 / 2}\left(\cos ^{2} \theta_{i+1}-\cos ^{2} \theta_{i}\right)-B_{i+1 / 2}\left(W_{i+1}-W_{i}\right)\right) \\
\pm & P_{i+1 / 2}\left(\mathcal{A}_{i+1 / 2}\left(W_{i+1}-W_{i}\right)-S_{1, i+1 / 2}\left(b_{i+1}-b_{i}\right)\right. \\
& +\left(S_{3, i+1 / 2}-S_{2, i+1 / 2}\right)\left(\cos ^{2} \theta_{i+1}-\cos ^{2} \theta_{i}\right)+ \\
& \left.\left.+S_{4, i+1 / 2}\left(\cos \theta_{i+1}-\cos \theta_{i}\right)-T_{i+1 / 2} \Delta x\right)\right\}
\end{aligned}
$$

The matrices appearing in the definition of the numerical scheme can be written as follows: 


$$
\mathcal{A}_{i+1 / 2}=\left[\begin{array}{c|c}
J_{i+1 / 2}^{1} & -B_{i+1 / 2}^{1,2} \\
\hline-B_{i+1 / 2}^{2,1} & J_{i+1 / 2}^{2}
\end{array}\right], \quad B_{i+1 / 2}=\left[\begin{array}{c|c}
0 & B_{i+1 / 2}^{1,2} \\
\hline B_{i+1 / 2}^{2,1} & 0
\end{array}\right]
$$

where

$$
\begin{aligned}
& J_{i+1 / 2}^{1}=\left[\begin{array}{cc}
0 & \cos \theta_{i+1 / 2} \\
-\bar{U}_{1, i+1 / 2}^{2} \cos \theta_{i+1 / 2}+c_{1, i+1 / 2}^{2}\left(\cos ^{2} \theta\right)_{i+1 / 2} & 2 \bar{U}_{1, i+1 / 2} \cos \theta_{i+1 / 2}
\end{array}\right], \\
& J_{i+1 / 2}^{2}=\left[\begin{array}{cc}
0 & \cos \theta_{i+1 / 2} \\
-\bar{U}_{2, i+1 / 2}^{2} \cos \theta_{i+1 / 2}+\Lambda_{2} c_{2, i+1 / 2}^{2}\left(\cos ^{2} \theta\right)_{i+1 / 2} & 2 \bar{U}_{2, i+1 / 2} \cos \theta_{i+1 / 2}
\end{array}\right], \\
& B_{i+1 / 2}^{1,2}=\left[\begin{array}{cc}
0 & 0 \\
-c_{1, i+1 / 2}^{2}\left(\cos ^{2} \theta\right)_{i+1 / 2} & 0
\end{array}\right], B_{i+1 / 2}^{2,1}=\left[\begin{array}{cc}
0 & 0 \\
-r \Lambda_{1} c_{2, i+1 / 2}^{2}\left(\cos ^{2} \theta\right)_{i+1 / 2} & 0
\end{array}\right] \text {, } \\
& S_{1, i+1 / 2}=\left[\begin{array}{c}
0 \\
-g H_{1, i+1 / 2} \cos \theta_{i+1 / 2} \\
0 \\
-g H_{2, i+1 / 2} \cos \theta_{i+1 / 2}
\end{array}\right], \\
& S_{2, i+1 / 2}=\left[\begin{array}{c}
0 \\
-g \frac{H_{1, i+1 / 2}}{2}\left(\frac{H_{1, i+1 / 2}}{2}+2 H_{2, i+1 / 2}\right) \cos \theta_{i+1 / 2} \\
0 \\
-g \frac{H_{2, i+1 / 2}}{2}\left(\frac{H_{2, i+1 / 2}}{2}+2 r \Lambda_{1} H_{1, i+1 / 2}\right) \cos \theta_{i+1 / 2}
\end{array}\right] \text {, } \\
& S_{3, i+1 / 2}=\left[\begin{array}{c}
0 \\
\frac{3 g}{4} H_{1, i+1 / 2}^{2} \cos \theta_{i+1 / 2} \\
0 \\
\frac{3 g}{4} \Lambda_{2} H_{2, i+1 / 2}^{2} \cos \theta_{i+1 / 2}
\end{array}\right], \quad S_{4, i+1 / 2}=\left[\begin{array}{c}
Q_{1, i+1 / 2} \\
Q_{1, i+1 / 2}^{2} / H_{1, i+1 / 2} \\
Q_{2, i+1 / 2} \\
Q_{2, i+1 / 2}^{2} / H_{2, i+1 / 2}
\end{array}\right] \text {, } \\
& T_{i+1 / 2}=\left[\begin{array}{c}
0 \\
0 \\
0 \\
\mathcal{T}_{i+1 / 2} / \cos \theta_{i+1 / 2}
\end{array}\right],
\end{aligned}
$$

where

$$
\mathcal{T}_{i+1 / 2}= \begin{cases}\mathcal{T}_{1, i+1 / 2}+\mathcal{T}_{2, i+1 / 2} & \text { if }\left|Q_{2, i+1 / 2}\right|>\frac{\Delta t \sigma_{c, i+1 / 2}}{\cos \theta_{i+1 / 2}} \\ \tau_{c r i t, i+1 / 2} & \text { otherwise }\end{cases}
$$

with

$$
\begin{gathered}
\mathcal{T}_{1, i+1 / 2}=-c_{2, i+1 / 2}^{2} \cos \theta_{i+1 / 2}(1-r) \operatorname{SGN}\left(\bar{U}_{2, i+1 / 2}\right) \tan \left(\delta_{0}\right), \\
\mathcal{T}_{2, i+1 / 2}=-H_{2, i+1 / 2} \bar{U}_{2, i+1 / 2}^{2} \frac{\sin \theta_{i+1}-\sin \theta_{i}}{\Delta x} \operatorname{SGN}\left(\bar{U}_{2, i+1 / 2}\right) \tan \left(\delta_{0}\right), \\
\sigma_{c, i+1 / 2}=(1-r) c_{2, i+1 / 2}^{2} \cos \theta_{i+1 / 2} \tan \left(\delta_{0}\right),
\end{gathered}
$$




$$
\begin{gathered}
\tau_{c r i t, i+1 / 2}=c_{2, i+1 / 2}^{2} \cos \theta_{i+1 / 2}\left(\left(\Lambda_{2}-r \Lambda_{1}\right) \frac{b_{i+1}-b_{i}+H_{2, i+1} \cos ^{2} \theta_{i+1}-H_{2, i} \cos ^{2} \theta_{i}}{\Delta x}+\right. \\
\left.+\left(1-\Lambda_{2}\right)\left(\frac{b_{i+1}-b_{i}}{\Delta x}+\frac{H_{2, i+1 / 2}}{4} \frac{\cos ^{2} \theta_{i+1}-\cos ^{2} \theta_{i}}{\Delta x}\right)\right) .
\end{gathered}
$$

In (73)-(80), we use the definitions

$$
\begin{gathered}
c_{k, 1+1 / 2}=\sqrt{g H_{k, i+1 / 2} \cos \theta_{i+1 / 2}}, \\
\bar{U}_{k, i+1 / 2}=\frac{\sqrt{H_{k, i}} \bar{U}_{k, i}+\sqrt{H_{k, i+1}} \bar{U}_{k, i+1}}{\sqrt{H_{k, i}}+\sqrt{H_{k, i+1}}},
\end{gathered}
$$

and

$$
\begin{aligned}
H_{k, i+1 / 2}=\frac{H_{k, i}+H_{k, i+1}}{2}, k & =1,2, \quad \cos \theta_{i+1 / 2}=\frac{\cos \theta_{i}+\cos \theta_{i+1}}{2}, \\
\left(\cos ^{2} \theta\right)_{i+1 / 2} & =\frac{\cos ^{2} \theta_{i}+\cos ^{2} \theta_{i+1}}{2},
\end{aligned}
$$

as well as the upwinded matrices

$$
P_{i+1 / 2}=\mathcal{K}_{i+1 / 2}\left(\operatorname{SGN}\left(D_{i+1 / 2}\right)\right) \mathcal{K}_{i+1 / 2}^{-1} .
$$

Here, if $D_{i+1 / 2}$ is a diagonal matrix defined by the eigenvalues of the matrix $\mathcal{A}_{i+1 / 2}, \mathcal{K}_{i+1 / 2}$ is the matrix whose columns are the associated eigenvectors. Let us denote by $\lambda_{j, i+1 / 2}$, $j=1, . ., 4$, the eigenvalues of matrix $\mathcal{A}_{i+1 / 2}$, then

$$
\operatorname{SGN}\left(D_{i+1 / 2}\right)=\left[\begin{array}{llll}
\operatorname{sgn}\left(\lambda_{1, i+1 / 2}\right) & & & \\
& \operatorname{sgn}\left(\lambda_{2, i+1 / 2}\right) & & \\
& & \operatorname{sgn}\left(\lambda_{3, i+1 / 2}\right) & \\
& & \operatorname{sgn}\left(\lambda_{4, i+1 / 2}\right)
\end{array}\right]
$$

\section{- Second step.}

We define

$$
W_{i}^{n+1}=\left[H_{1, i}^{*} Q_{1, i}^{*} H_{2, i}^{*} Q_{2, i}^{n+1}\right]^{T}
$$

and

$$
Q_{2, i}^{n+1}= \begin{cases}Q_{2, i}^{*}+\left(\mathcal{T}_{1, i}^{*}+\mathcal{T}_{2, i}^{*}\right) \Delta t & \text { if }\left|Q_{2, i}^{*}\right|>\frac{\sigma_{c, i}^{*} \Delta t}{\cos \theta_{i}} \\ 0 & \text { otherwhise }\end{cases}
$$

with

$$
\begin{gathered}
\mathcal{T}_{1, i}^{*}=-(1-r) \frac{\left(c_{2, i-1 / 2}^{*}\right)^{2}+\left(c_{2, i+1 / 2}^{*}\right)^{2}}{2} \cos \theta_{i} \operatorname{SGN}\left(Q_{2, i}^{*}\right) \tan \left(\delta_{0}\right), \\
\mathcal{T}_{2, i}^{*}=-\frac{H_{2, i-1 / 2}^{*}+H_{2, i+1 / 2}^{*}}{2}\left(\bar{U}_{2, i}^{*}\right)^{2} \frac{\sin \theta_{i+1 / 2}-\sin \theta_{i-1 / 2}}{\Delta x} \operatorname{SGN}\left(Q_{2, i}^{*}\right) \tan \left(\delta_{0}\right),
\end{gathered}
$$

in which

$$
\sigma_{c, i}^{*}=(1-r) \frac{\left(c_{2, i-1 / 2}^{*}\right)^{2}+\left(c_{2, i+1 / 2}^{*}\right)^{2}}{2} \cos \theta_{i} \tan \left(\delta_{0}\right)
$$

with

$$
c_{2, i+1 / 2}^{*}=\sqrt{g \frac{H_{2, i}^{*}+H_{2, i+1}^{*}}{2} \cos \theta_{i+1 / 2}} .
$$


The definition of $Q_{2, i}^{n+1}$ proposed in equation (84), is based on the numerical treatment of Coulomb friction term introduced by Mangeney et al. in [31]. Observe that the definition of the Coulomb term, implies that if $|T|<\sigma_{c}$ then $Q_{2}=0$. A way to impose implicitely this definition in the numerical scheme is the one proposed by equation (84).

Remark 11 Observe that

$$
\frac{S_{3, i+1 / 2}\left(\cos ^{2} \theta_{i+1}-\cos ^{2} \theta i\right)+S_{4, i+1 / 2}\left(\cos \theta_{i+1}-\cos \theta_{i}\right)}{\Delta x}
$$

is a second order approximation of $\left.\partial_{\theta} F d_{x} \theta\right|_{x=x_{i+1 / 2}}$.

This numerical scheme could be seen as a predictor-corrector numerical scheme for the Coulomb friction term. In the first step, the term $\mathcal{T}_{i+1 / 2}$ is only considered in the uncentered part of the numerical scheme. Note that in the definition of $\mathcal{T}_{i+1 / 2}$ (see (79)) a second order approximation of the Coulomb friction term is considered if $\left|Q_{2, i+1 / 2}\right|>\frac{\Delta t \sigma_{c, i+1 / 2}}{\cos \theta_{i+1 / 2}}$. Otherwise, we set $\mathcal{T}_{i+1 / 2}=$ $\tau_{c r i t, i+1 / 2}$, that is also a second order approximation of the value of the Coulomb friction term in order that all terms in the last equation of system (65) are balanced taking into account $U_{2}=0$. This relation is critical in order to obtain a well-balanced numerical scheme for the solutions corresponding to water at rest and no movement of the sediment in the model.

After this first step, a predicted value $Q_{2, i}^{*}$ is computed and then, following [31], the final value $Q_{2, i}^{n+1}$ is computed using (84).

Concerning the stability requirements, we use the following CFL-condition

$$
\max \left\{\left\|D_{i+1 / 2}\right\|_{\infty}, 0 \leq i \leq M\right\} \frac{\Delta t}{\Delta x} \leq \gamma,
$$

where $0<\gamma \leq 1$, and $M$ is the number of cells into which the space domain is decomposed.

We have the following result:

THEOREM 2 The previous numerical scheme verifies the following properties:

i) The numerical scheme preserves all the stationary solutions satisfying

$$
\begin{gathered}
\bar{U}_{1}=\bar{U}_{2}=0, \\
b+\left(H_{1}+H_{2}\right) \cos ^{2} \theta=c s t,
\end{gathered}
$$

and

$$
\left|\left(\Lambda_{2}-r \Lambda_{1}\right) \partial_{x}\left(b+H_{2} \cos ^{2} \theta\right)+\left(1-\Lambda_{2}\right)\left(\partial_{x} b+\frac{H_{2}}{4} \partial_{x} \cos ^{2} \theta\right)\right| \leq(1-r) \tan \left(\delta_{0}\right),
$$

such as

$$
\begin{gathered}
\mid\left(\Lambda_{2}-r \Lambda_{1}\right)\left(b\left(x_{i+1}\right)-b\left(x_{i}\right)+H_{2}\left(x_{i+1}\right) \cos ^{2}\left(\theta\left(x_{i+1}\right)\right)-H_{2}\left(x_{i}\right) \cos \left(\theta\left(x_{i}\right)\right)\right)+ \\
+\left(1-\Lambda_{2}\right)\left(b\left(x_{i+1}\right)-b\left(x_{i}\right)+\frac{H_{2}\left(x_{i+1}\right)+H_{2}\left(x_{i}\right)}{4}\left(\cos ^{2}\left(\theta\left(x_{i+1}\right)\right)-\cos ^{2}\left(\theta\left(x_{i}\right)\right)\right)\right) \mid \leq \\
\leq(1-r) \tan \left(\delta_{0}\right) \Delta x .
\end{gathered}
$$

ii) The numerical scheme preserves all stationary solutions up to order 2. 
PROOF.-

Using the definition of the Roe matrix $\mathcal{A}_{i+1 / 2}$ it is easy to prove that

$$
\begin{gathered}
\mathcal{A}_{i+1 / 2}\left(W_{i+1}-W_{i}\right)=\mathcal{F}\left(\cos \theta_{i+1 / 2},\left(\cos ^{2} \theta\right)_{i+1 / 2}, W_{i+1}\right)-\mathcal{F}\left(\cos \theta_{i+1 / 2},\left(\cos ^{2} \theta\right)_{i+1 / 2}, W_{i}\right)- \\
-B_{i+1 / 2}\left(W_{i+1}-W_{i}\right) .
\end{gathered}
$$

Observe that the terms that are multiplied with $P_{i+1 / 2}$ in (73) are equal to the centered components of $\mathcal{D} \mathcal{F}_{i+1 / 2}^{ \pm}$defined by (88) except the Coulomb friction term $T_{i+1 / 2} \Delta x$. That is, using (87), we could rewrite the numerical fluxes as

$$
\mathcal{D} \mathcal{F}_{i+1 / 2}^{ \pm}=\frac{1}{2}\left\{R_{1, i+1 / 2} \pm P_{i+1 / 2} R_{2, i+1 / 2}\right\},
$$

where,

$$
R_{2, i+1 / 2}=R_{1, i+1 / 2}-T_{i+1 / 2} \Delta x
$$

and $R_{1, i+1 / 2}$ is defined by

$$
\begin{gathered}
R_{1, i+1 / 2}=\mathcal{F}\left(\cos \theta_{i+1 / 2},\left(\cos ^{2} \theta\right)_{i+1 / 2}, W_{i+1}\right)-\mathcal{F}\left(\cos \theta_{i+1 / 2},\left(\cos ^{2} \theta\right)_{i+1 / 2}, W_{i}\right)+ \\
\quad+S_{3, i+1 / 2}\left(\cos ^{2} \theta_{i+1}-\cos ^{2} \theta_{i}\right)+S_{4, i+1 / 2}\left(\cos \theta_{i+1}-\cos \theta_{i}\right)- \\
-S_{1, i+1 / 2}\left(b_{i+1}-b_{i}\right)-S_{2, i+1 / 2}\left(\cos ^{2} \theta_{i+1}-\cos ^{2} \theta_{i}\right)-B_{i+1 / 2}\left(W_{i+1}-W_{i}\right) .
\end{gathered}
$$

Let us prove $i$ ). Observe that in this case the first and third components of $R_{1, i+1 / 2}$ are equal to zero. The second component of $R_{1, i+1 / 2}$ is equal to

$$
\begin{gathered}
{\left[R_{1, i+1 / 2}\right]_{2}=g \frac{H_{1, i+1}^{2}}{2} \cos \theta_{i+1 / 2}\left(\cos ^{2} \theta\right)_{i+1 / 2}-g \frac{H_{1, i}^{2}}{2} \cos \theta_{i+1 / 2}\left(\cos ^{2} \theta\right)_{i+1 / 2}+} \\
+\frac{3}{4} g H_{1, i+1 / 2}^{2} \cos \theta_{i+1 / 2}\left(\cos ^{2} \theta_{i+1}-\cos ^{2} \theta_{i}\right)+g H_{1, i+1 / 2} \cos \theta_{i+1 / 2}\left(b_{i+1}-b_{i}\right)+ \\
+g \frac{H_{1, i+1 / 2}}{2}\left(\frac{H_{1, i+1 / 2}}{2}+2 H_{2, i+1 / 2}\right) \cos \theta_{i+1 / 2}\left(\cos ^{2} \theta_{i+1}-\cos ^{2} \theta_{i}\right)+ \\
+g H_{1, i+1 / 2} \cos \theta_{i+1 / 2}\left(\cos ^{2} \theta\right)_{i+1 / 2}\left(H_{2, i+1}-H_{2, i}\right) .
\end{gathered}
$$

We can write

$$
\begin{gathered}
g \frac{H_{1, i+1}^{2}}{2} \cos \theta_{i+1 / 2}\left(\cos ^{2} \theta\right)_{i+1 / 2}-g \frac{H_{1, i}^{2}}{2} \cos \theta_{i+1 / 2}\left(\cos ^{2} \theta\right)_{i+1 / 2}= \\
=g \cos \theta_{i+1 / 2} H_{1, i+1 / 2}\left(\cos ^{2} \theta\right)_{i+1 / 2}\left(H_{1, i+1}-H_{1, i}\right)
\end{gathered}
$$

and then obtain

$$
\begin{aligned}
{\left[R_{1, i+1 / 2}\right]_{2}=} & g \cos \theta_{i+1 / 2} H_{1, i+1 / 2}\left\{\left(\cos ^{2} \theta\right)_{i+1 / 2}\left(H_{2, i+1}+H_{1, i+1}-\left(H_{2, i}+H_{1, i}\right)\right)+\right. \\
& \left.+g\left(H_{1, i+1 / 2}+H_{2, i+1 / 2}\right)\left(\cos ^{2} \theta_{i+1}-\cos ^{2} \theta_{i}\right)+b_{i+1}-b_{i}\right\} .
\end{aligned}
$$

Thanks to the definition of $\left(\cos ^{2} \theta\right)_{i+1 / 2}=\left(\cos ^{2} \theta_{i}+\cos ^{2} \theta_{i+1}\right) / 2$, we can use in the previous equation the following rule (discrete version of the derivative of a product),

$$
a b-c d=\frac{a+c}{2}(b-d)+(a-c) \frac{b+d}{2}, \quad \forall a, b, c, d \in \mathcal{R},
$$

to obtain

$$
\left[R_{1, i+1 / 2}\right]_{2}=g \cos \theta_{i+1 / 2} H_{1, i+1 / 2}\left\{\left(b_{i+1}+H_{1, i+1} \cos ^{2} \theta_{i+1}+H_{2, i+1} \cos ^{2} \theta_{i+1}\right)-\right.
$$




$$
\left.-\left(b_{i}+H_{1, i} \cos ^{2} \theta_{i}+H_{2, i} \cos ^{2} \theta_{i}\right)\right\} .
$$

For $i$ ) we have constant free surface, $b+H_{1} \cos ^{2} \theta+H_{2} \cos ^{2} \theta=c s t$, then the second component of $R_{1, i+1 / 2}$ is equal to zero.

The fourth component of $R_{1, i+1 / 2}$ is equal to

$$
\begin{gathered}
{\left[R_{1, i+1 / 2}\right]_{4}=g \cos \theta_{i+1 / 2} H_{2, i+1 / 2}\left(\Lambda_{2}\left(b_{i+1}-b_{i}\right)+\Lambda_{2}\left(\cos ^{2} \theta\right)_{i+1 / 2}\left(H_{2, i+1}-H_{2, i}\right)+\right.} \\
\left.+\Lambda_{2} H_{2, i+1 / 2}\left(\cos ^{2} \theta_{i+1}-\cos ^{2} \theta_{i}\right)+r \Lambda_{1}\left(H_{1, i+1 / 2}\left(\cos ^{2} \theta_{i+1}-\cos ^{2} \theta_{i}\right)+\left(\cos ^{2} \theta\right)_{i+1 / 2}\left(H_{1, i+1}-H_{1, i}\right)\right)\right)+ \\
+\left(1-\Lambda_{2}\right)\left(g \frac{H_{2, i+1 / 2}^{2}}{4} \cos \theta_{i+1 / 2}\left(\cos ^{2} \theta_{i+1}-\cos ^{2} \theta_{i}\right)+g H_{2, i+1 / 2} \cos \theta_{i+1 / 2}\left(b_{i+1}-b_{i}\right)\right) .
\end{gathered}
$$

Using (89) and $b+H_{2} \cos ^{2} \theta+H_{1} \cos ^{2} \theta=c s t$ we obtain

$$
\begin{gathered}
{\left[R_{1, i+1 / 2}\right]_{4}=g \cos \theta_{i+1 / 2} H_{2, i+1 / 2}\left(\Lambda_{2}-r \Lambda_{1}\right)\left(b_{i+1}-b_{i}+H_{2, i+1} \cos ^{2} \theta_{i+1}-H_{2, i} \cos ^{2} \theta_{i}\right)+} \\
+\left(1-\Lambda_{2}\right) g \cos \theta_{i+1 / 2} H_{2, i+1 / 2}\left(\frac{H_{2, i+1 / 2}}{4}\left(\cos ^{2} \theta_{i+1}-\cos ^{2} \theta_{i}\right)+b_{i+1}-b_{i}\right) .
\end{gathered}
$$

Finally, we conclude that the three first components of $R_{1, i+1 / 2}$ are zero.

For the fourth component observe that $\left[R_{1, i+1 / 2}\right]_{4}$ exactly coincides with $\left(\mathcal{T}_{\text {crit }, i+1 / 2} \Delta x / \cos \theta_{i+1 / 2}\right)$ where $\mathcal{T}_{\text {crit }, i+1 / 2}$ is defined by (80). Moreover, by hypothesis the given stationary solution verifies $Q_{2, i}^{n}=0$, and by (79) we obtain

$$
\left[T_{i+1 / 2}\right]_{4}=\frac{\mathcal{T}_{c r i t, i+1 / 2}}{\cos \theta_{i+1 / 2}}
$$

Then,

$$
\left[R_{2, i+1 / 2}\right]_{4}=\left[R_{1, i+1 / 2}\right]_{4}-\left[T_{i+1 / 2}\right]_{4} \Delta x=0, \quad \Rightarrow \quad R_{2, i+1 / 2}=0 .
$$

So, $\mathcal{D} \mathcal{F}_{i+1 / 2}^{ \pm}=R_{1, i+1 / 2}$. Additionally, in the second step we have $H_{1, i}^{n+1}=H_{1, i}^{n}, Q_{1, i}^{n+1}=Q_{1, i}^{n}$, $H_{2, i}^{n+1}=H_{2, i}^{n}$. Moreover, by (72) we deduce

$$
\begin{gathered}
Q_{2, i}^{*}=-\frac{\Delta t}{\Delta x} \frac{R_{1, i+1 / 2}+R_{1, i-1 / 2}}{2}= \\
=-\frac{\Delta t}{2 \Delta x}\left(\left(\Lambda_{2}-r \Lambda_{1}\right) g H_{2, i+1 / 2} \cos \theta_{i+1 / 2}\left\{\left(b_{i+1}+H_{2, i+1} \cos ^{2} \theta_{i+1}\right)-\left(b_{i}+H_{2, i} \cos ^{2} \theta_{i}\right)\right\}+\right. \\
\left.+\left(\Lambda_{2}-\Lambda_{1} r\right) g H_{2, i-1 / 2} \cos \theta_{i-1 / 2}\left\{\left(b_{i}+H_{2, i} \cos ^{2} \theta_{i}\right)-\left(b_{i-1}+H_{2, i-1} \cos ^{2} \theta_{i-1}\right)\right\}\right)+ \\
+\left(1-\Lambda_{2}\right) g \cos \theta_{i+1 / 2} H_{2, i+1 / 2}\left(\frac{H_{2, i+1 / 2}}{4}\left(\cos ^{2} \theta_{i+1}-\cos ^{2} \theta_{i}\right)+b_{i+1}-b_{i}\right)+ \\
+\left(1-\Lambda_{2}\right) g \cos \theta_{i-1 / 2} H_{2, i-1 / 2}\left(\frac{H_{2, i-1 / 2}}{4}\left(\cos ^{2} \theta_{i}-\cos ^{2} \theta_{i-1}\right)+b_{i}-b_{i-1}\right)
\end{gathered}
$$

Then, by (86)

$$
\left|Q_{2, i}^{*}\right| \leq \Delta t(1-r) g \frac{H_{2, i+1 / 2} \cos \theta_{i+1 / 2}+H_{2, i-1 / 2} \cos \theta_{i-1 / 2}}{2} \tan \left(\delta_{0}\right)=\frac{\sigma_{c, i}^{*} \Delta t}{\cos \theta_{i}},
$$

where $\sigma_{c, i}^{*}$ is defined by (85). Then, by (84) we obtain $Q_{2, i}^{n+1}=0$. Finally, we conclude that the stationary solutions verifying (52), (53), (54) satisfying (86) are exactly preserved. 
To prove $i$ ) it is enough to observe that any stationary solution verifies

$$
\partial_{W} F \partial_{x} W+\partial_{\theta} F \partial_{x} \theta-G_{1}-G_{2}-B(W) \partial_{x} W-T=0 .
$$

Moreover, $\mathcal{D} \mathcal{F}_{i+1 / 2}^{ \pm}=\frac{1}{2}\left\{R_{1, i+1 / 2} \pm P_{i+1 / 2} R_{2, i+1 / 2}\right\}$ and $R_{2, i+1 / 2}$ is a second order approximation of (91). Finally, in the second step a second order approximation of $T$ is added to $R_{1}$; so finally we have another second order approximation of (91) and we can conclude that $W_{i}^{n+1}=W_{i}^{n}+\mathcal{O}\left(\Delta x^{2}\right)$.

\section{$5 \quad$ Numerical tests}

In the following numerical tests the parameters are set to: $K=1, K_{i n}=0$, and the ratio of densities $r=0.2$.

Different situations of wet/dry fronts appear in the numerical tests. We use here the numerical treatment proposed by Castro et al. in [10]. It basically consists in a local redefinition of the topography in the inter-cells corresponding to wet/dry transitions in order to avoid the generation of spurious pressure forces.

\subsection{Submarine landslide}

The numerical test presented here is devoted to the simulation of a submarine landslide into a rectangular channel of $10 \mathrm{~m}$ length. The topography is given by a strait line whose slope is set equal to 0.2 (i. e. slope angle $\theta=11.31^{\circ}$ ). The Coulomb friction angle is set equal to $\delta_{0}=25^{\circ}$. Finally, the CFL parameter is set to 0.8 .

As initial conditions we set $\bar{U}_{1}(x)=\bar{U}_{2}(x)=0, \eta_{1}(x)=b(x)+\left(H_{1}(x)+H_{2}(x)\right) \cos ^{2} \theta$ is constant and equals 2.7 (see Figure $3(\mathrm{a}))$ and

$$
H_{2}(x)= \begin{cases}1 / \cos \theta, & \text { if } 7 \leq x \leq 8 \\ 0, & \text { otherwise }\end{cases}
$$

Free boundary conditions are imposed at both channel ends.

Figure 3 shows the evolution of the submarine landslide from the initial condition until the mass stops on the slope and forms a deposit (see Figure $3(\mathrm{f})$ ) for $\Delta x=0.05$.

As consequence of the submarine landslide, some water waves are generated at the free surface (see Figures 3(b) and 3(c)). They travel along the channel and go away as shown in Figures $3(\mathrm{~d})-3(\mathrm{f})$.

In Figure 5.1 we compare the final stationary interface that we obtain for four different meshes with $\Delta x=1 / 20, \Delta x=1 / 40, \Delta x=1 / 80$ and $\Delta x=1 / 320$. Only some small differences near the "wet/dry" fronts can be observed, due to the numerical treatment of the wet/dry fronts. This effect is also present if we study for example the bilayer Shallow Water equations (see [10]).

\subsection{Landslide impinging a lake}

In this subsection two numerical experiments are presented. They simulate the waves caused in a lake by an avalanche of a granular material that slides along a plane of constant slope and that falls on it.

In both of them, a rectangular channel of $10 \mathrm{~m}$ length long is considered. The topography is given by

$$
b(x)= \begin{cases}1-\frac{x}{5}, & \text { if } 0 \leq x \leq 5 \\ 0, & \text { otherwise }\end{cases}
$$

With this definition the bed curvature is infinite at $x=5$, because the slopes are discontinuous. In this point the change of variables is not justified. Nevertheless, the resulting integrated system 


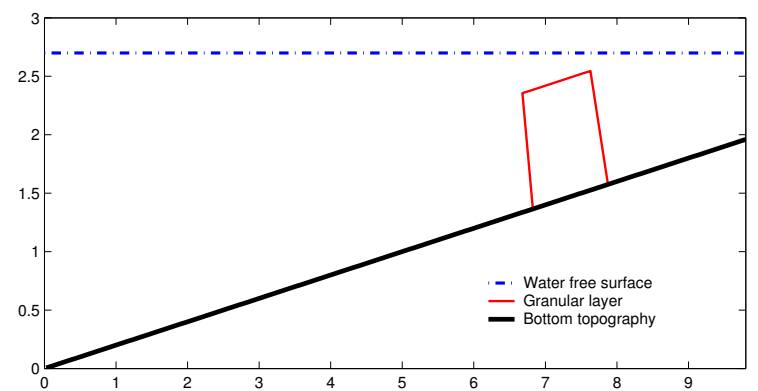

(a) Initial condition $0.0 \mathrm{~s}$.

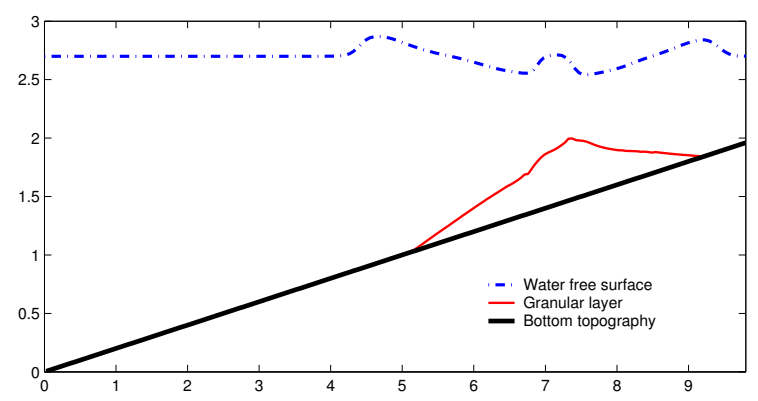

(c) $0.5 \mathrm{~s}$

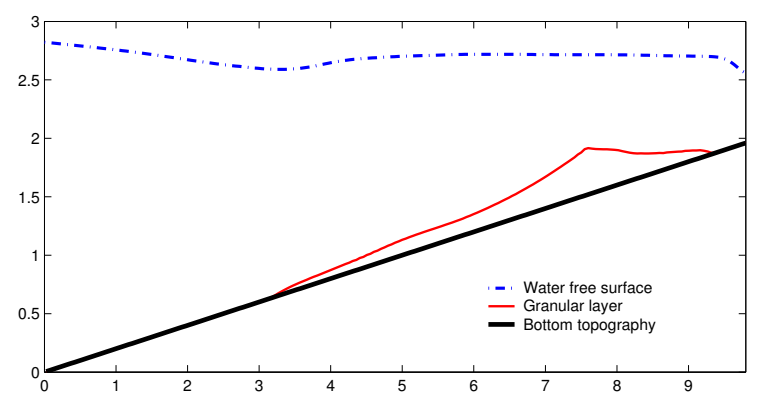

(e) $1.5 \mathrm{~s}$

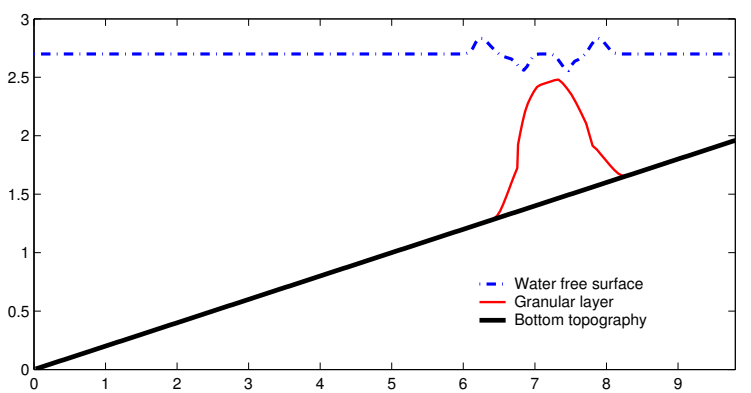

(b) $0.1 \mathrm{~s}$.

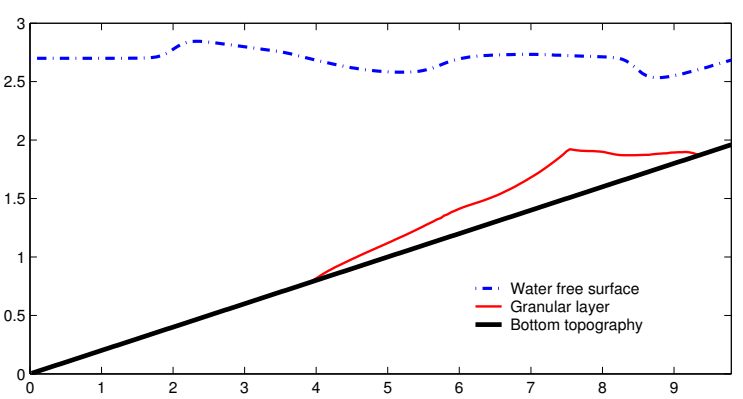

(d) $1.0 \mathrm{~s}$

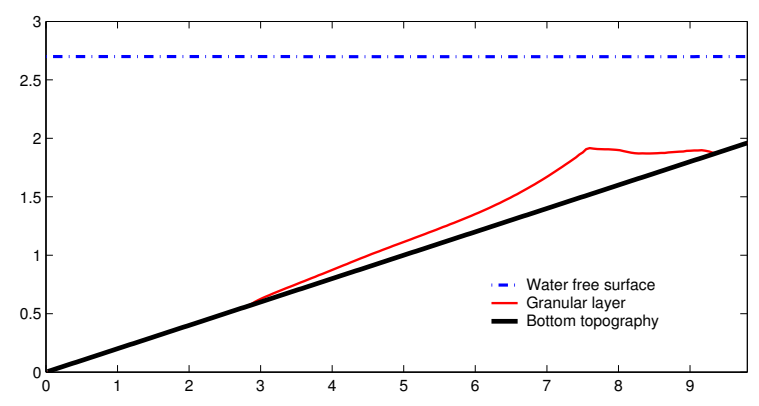

(f) $5 \mathrm{~s}$. Steady state (granular material)

Figure 3: Submarine landslide $(\Delta x=0.05)$ : water surface and landslide evolution (Horizontal $\mathrm{x}(\mathrm{m})$, Vertical $\mathrm{z}(\mathrm{m}))$ 


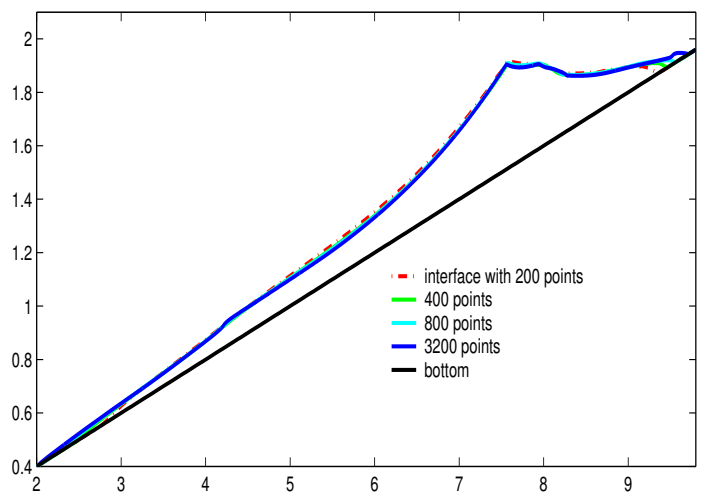

Figure 4: Submarine landslide: stationary landslide configuration for $\Delta x=1 / 20, \Delta x=1 / 40$, $\Delta x=1 / 80$ and $\Delta x=1 / 320$

has a solution, that can be approximated numerically. The solution of the system is the limit of the solutions obtained using a regularization of the bottom.

From a numerical point of view, the bed at this point can be seen as approximated by a parabola, because we use the middle point formula to approximate the derivatives (See Remark $11)$.

The CFL parameter is set equal to 0.8 , and $\Delta x=0.05$. As initial condition we set

$$
\begin{gathered}
\bar{U}_{1}(x)=0, \quad \bar{U}_{2}(x)=0, \\
H_{1}(x)= \begin{cases}(0.7-b(x)) / \cos ^{2} \theta, & \text { if } 1.5 \leq x \leq 2.5, \\
0, & \text { otherwise, }\end{cases}
\end{gathered}
$$

and

$$
H_{2}(x)= \begin{cases}(0.4-b(x)) / \cos ^{2} \theta, & \text { if } 3 \leq x \leq 5, \\ 0.4, & \text { otherwise }\end{cases}
$$

This initial condition simulates that, initially, the granular material is not submerged, and it is placed at $[1.5,2.5]$, while the water layer is placed at $x \geq 4$ (see Figures $5(\mathrm{a})$ and $7(\mathrm{a})$ ). Free boundary conditions are imposed at both channel ends.

In the first experiment considered here, the Coulomb friction angle is set equal to $\delta_{0}=10^{\circ}$, while the angle of the plane is equal to $11.31^{\circ}$. Therefore, all the granular material will slide down the plane and will deposit at the basin of the lake as it is shown in Figures 5(b)-5(e). The stationary state for the granular layer is shown (see Figure $5(\mathrm{f})$ ). When the landslide contacts the water, a wave at the free surface of the lake is generated and propagates in the same direction of the landslide as it is shown in Figures 5(b) and 5(c). Figures 5(d) and 5(e) show the generation and propagation of a shock at the interface of the granular material and the water, traveling in the opposite direction of the landslide propagation. Observe that the granular deposit is located at the bottom of the lake forming a smooth pile whose form is determined by the Coulomb angle and the flow history (see Figure 5(f)).

In Figure 5.2 we compare the stationary interface that we obtain for $\Delta x=1 / 20, \Delta x=1 / 40$, $\Delta x=1 / 80$ and $\Delta x=1 / 320$. As in previous test the convergence to a stationary profile is achieved.

In the second experiment, the Coulomb angle is set equal to $\delta_{0}=25^{\circ}$. The evolution of the landslide is different from the previous one. The main difference is the length of the avalanche: in this case, the granular material is not deposited in the basin of the lake as it is shown in Figures 7 (b)-7(f). As in the previous case, when the landslide contacts the water, a wave at the free surface of the lake is generated and propagated in the same direction of the landslide as it is shown in 


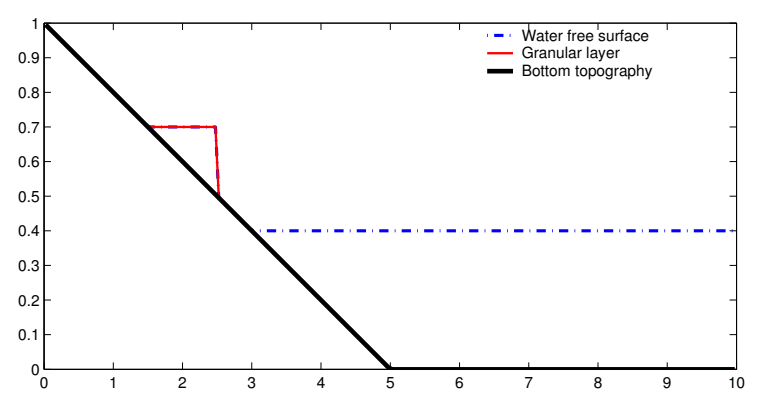

(a) $0.0 \mathrm{~s}$.

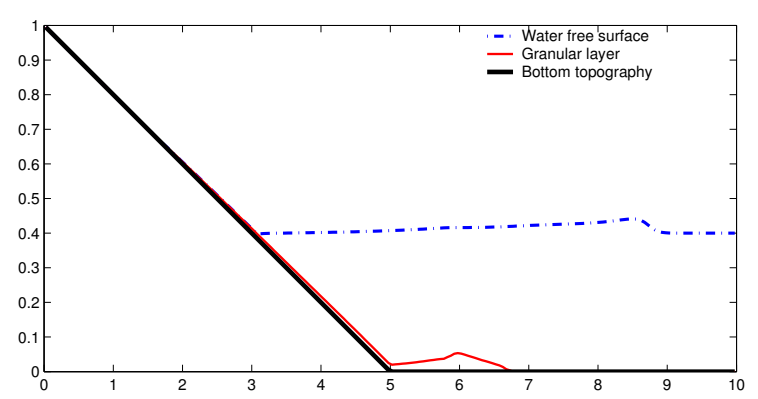

(c) $3.0 \mathrm{~s}$

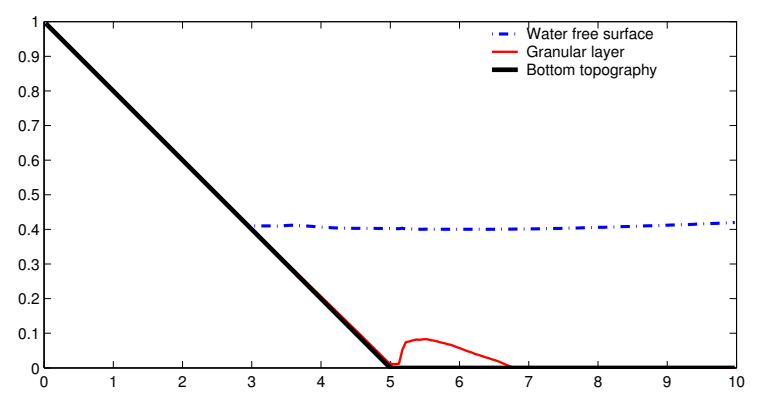

(e) $4.5 \mathrm{~s}$

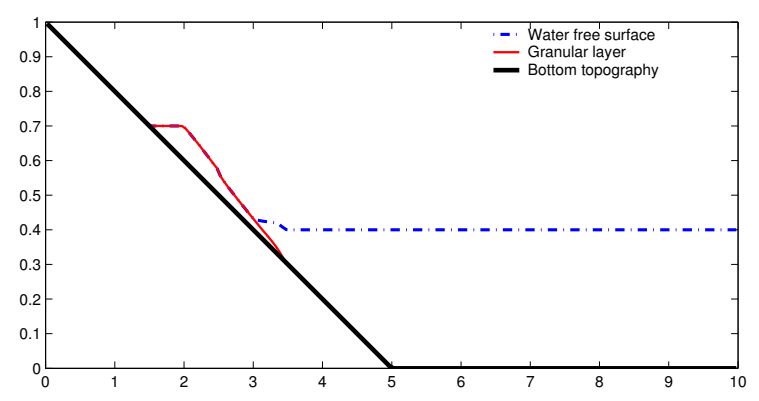

(b) $0.5 \mathrm{~s}$.

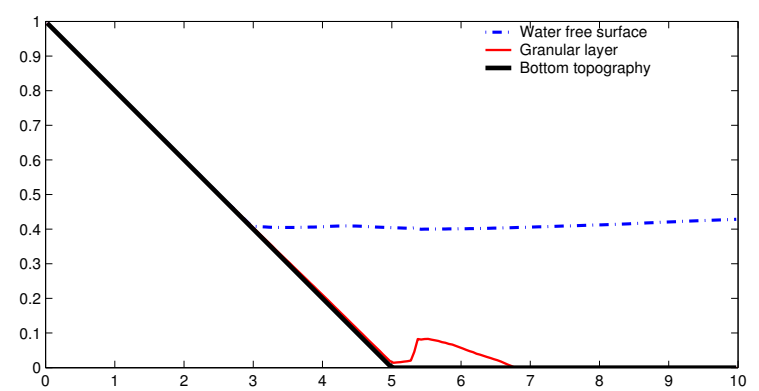

(d) $4.0 \mathrm{~s}$

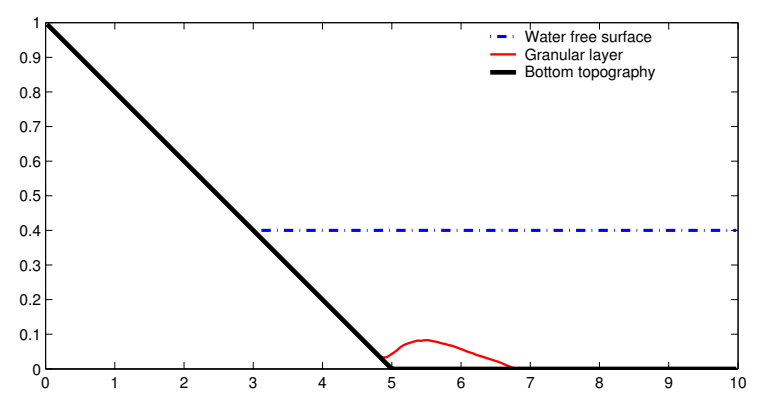

(f) Stationary state $\mathrm{s}$

Figure 5: Landslide impinging a lake (experiment 1): Water surface and landslide evolution. (Horizontal x $(\mathrm{m})$, Vertical $\mathrm{z}(\mathrm{m})$ ) 


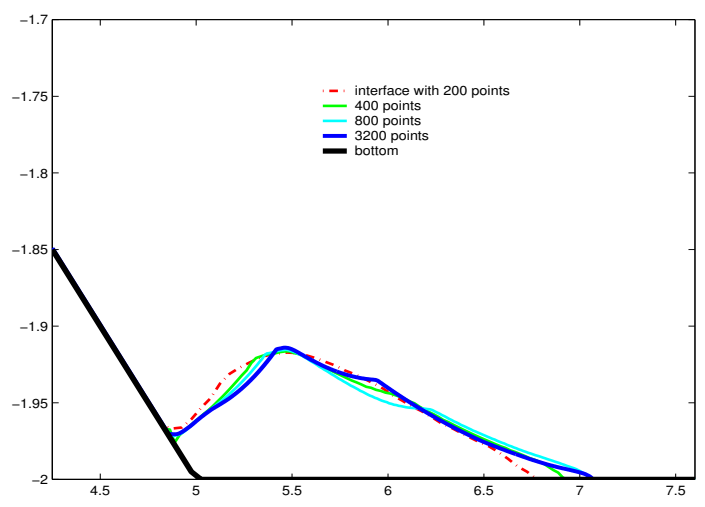

Figure 6: Landslide impinging a lake (experiment 1): stationary landslide configuration for $\Delta x=$ $1 / 20, \Delta x=1 / 40, \Delta x=1 / 80$ and $\Delta x=1 / 320$

Figures 7(b)- 7(d), with a wave amplitude that is smaller than in the previous experiment. Finally, the steady state for the granular material is shown in Figure $7(\mathrm{f})$.

In Figure 8 we show the stationary profiles of the landslide for different values of $\delta_{0}$. In Figure 8 (a) the results correspond to $\delta_{0}$ equals to $10,11.31$ (the angle of the bottom in [0,5]), 12 and 13 degrees. In the case of 13 degrees we obtain that the rock layer is not completely submerged. For previous values we always obtain that this layer is completely submerged.

In Figure 8(b) we show the stationary profiles of the landslide for $\delta_{0}$ equals to 15,20 and 25 degrees. In all cases we have a profile that is partially submerged.

Using the friction angle $\delta=15^{\circ}$, a layer of quasi-constant thickness of material remains on the subaerial as well as on the submarine part of the plane once the flow stops. This deposit seems to be in agreement with the experimental results of ([8], Figure 3). Indeed, they show that the minimum deposit thickness for which a flow is possible commonly called $h_{\text {stop }}$ is unchanged when the flow occurs either in water or in air.

\subsection{Tsunami generation experiment}

This numerical example is inspired by that presented in the paper of Heinrich et al. (see [24]), and the objective is to understand the main mechanisms of water wave generation and propagation produced by submarine landslides. To do that, a $30 \mathrm{~km}$ long domain is considered, where a simplified topography is considered given by $b(x)=2500-H(x)$ where:

$$
H(x)= \begin{cases}10+490 \exp \left(-6.1429 \cdot 10^{-4}(10000-x)\right), & \text { if } x \leq 10000 \\ 2500-2000 \exp \left(-1.5050 \cdot 10^{-4}(x-10000)\right), & \text { otherwise }\end{cases}
$$

As initial condition we imposed $\bar{U}_{i}(x)=0, i=1,2$,

$$
H_{2}(x)=\frac{1}{\cos ^{2} \theta} \max \left(H(x)-\left(500+\frac{(x-10000)^{2}}{1.8 \cdot 10^{4}}\right), 0\right), \text { and } H_{1}(x)=\frac{H(x)}{\cos ^{2} \theta}-H_{2}(x) .
$$

Figure 9(a) shows the initial condition. The Coulomb friction angle is set to $\delta_{0}=12^{\circ}$ (as in [24]). The CFL condition is equal to 0.8. We use a mesh composed of 500 cells. Free free boundary conditions are imposed at $x=0$ and $x=30000$. With this data the experiment evolves during 600 s.

Figure 10 shows the generation and propagation of the tsunami wave. Observe that, water ahead of the front face of the slide is pushed away, creating a positive wave in the slide direction 


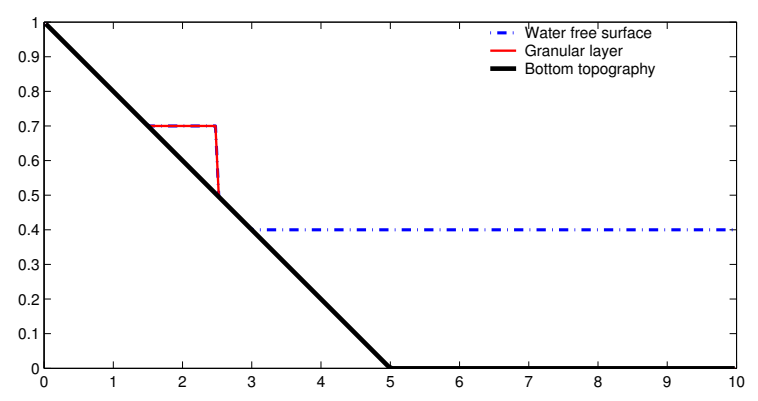

(a) $0.0 \mathrm{~s}$.

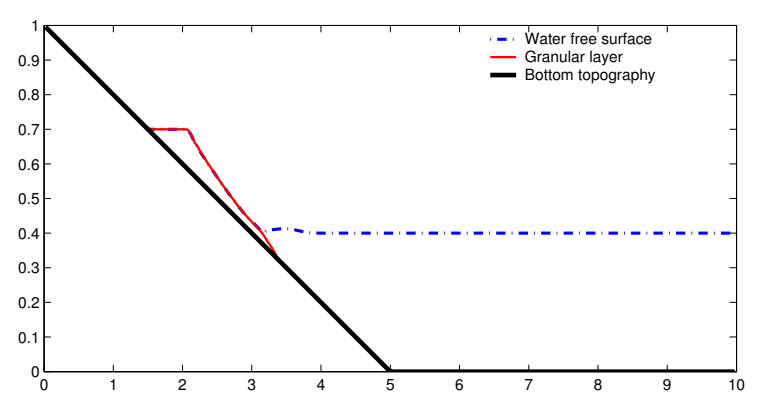

(c) $1.0 \mathrm{~s}$

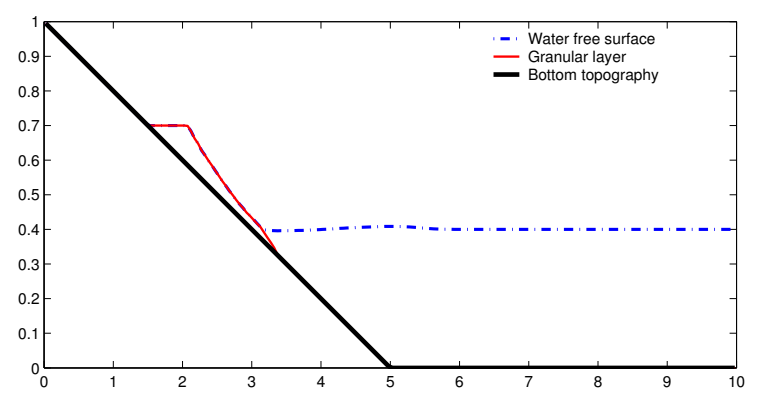

(e) $2.0 \mathrm{~s}$

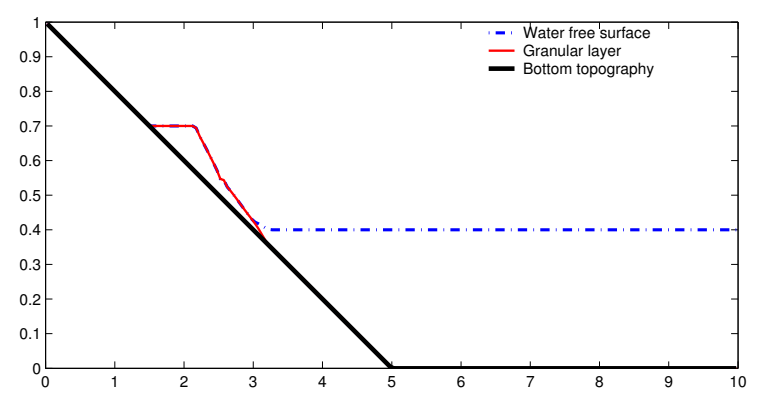

(b) $0.5 \mathrm{~s}$.

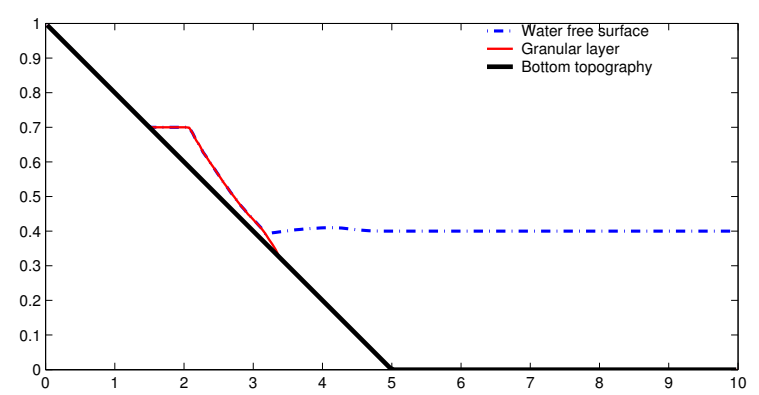

(d) $1.5 \mathrm{~s}$

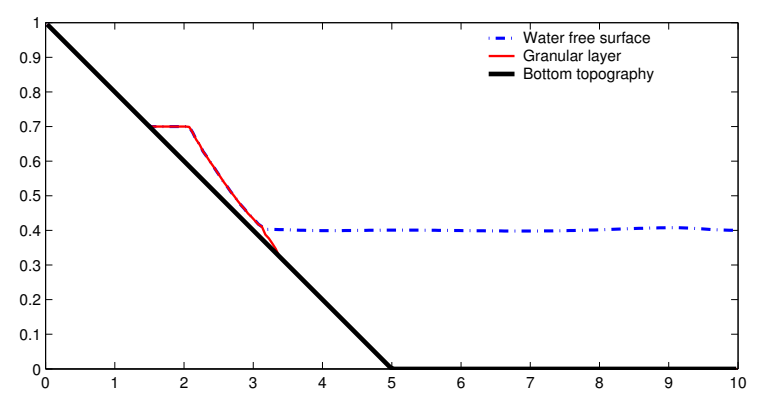

(f) $4.0 \mathrm{~s}$

Figure 7: Landslide impinging a lake (experiment 2): Water surface and landslide evolution. (Horizontal x $(\mathrm{m})$, Vertical $\mathrm{z}(\mathrm{m})$ ) 


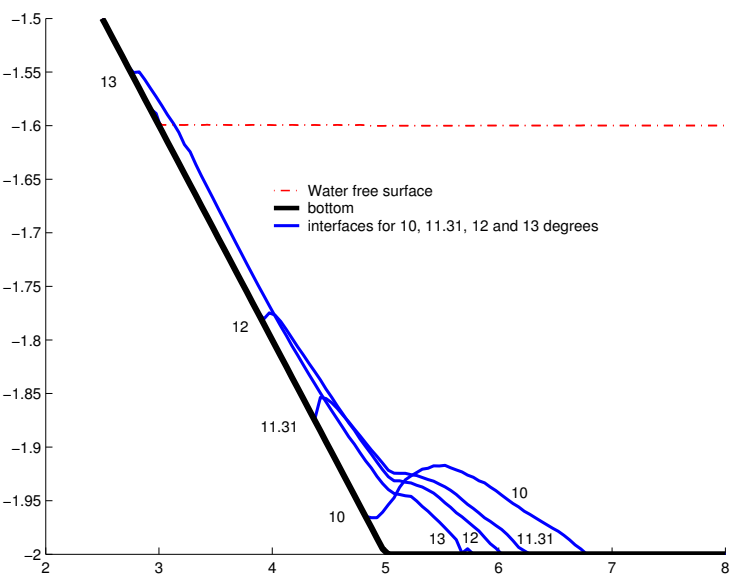

(a) Stationary landslide profiles for $\delta_{0}$ equals to 10 , 11.31 (angle of the plane), 12 and 13 degrees

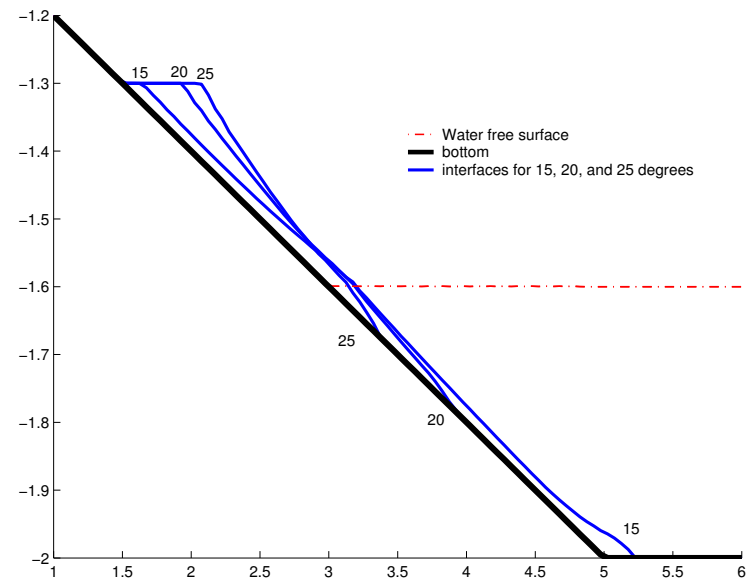

(b) Statinary landslide profiles for $\delta_{0}$ equal to 15,20 and 25 degrees

Figure 8: Landslide impinging a lake: Comparison of stationary landslide profiles for different values of $\delta_{0}$.

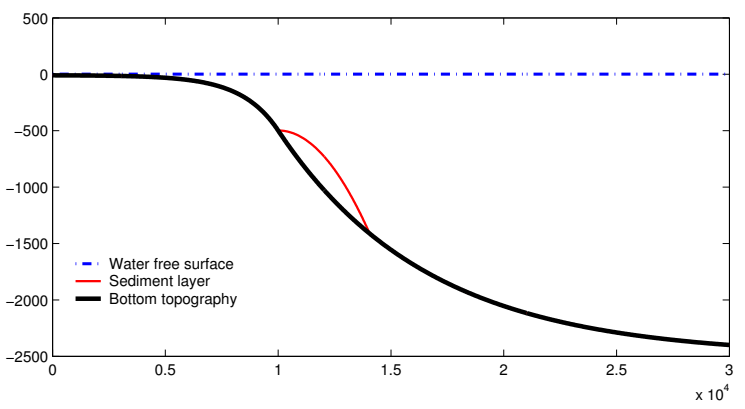

(a) Initial condition

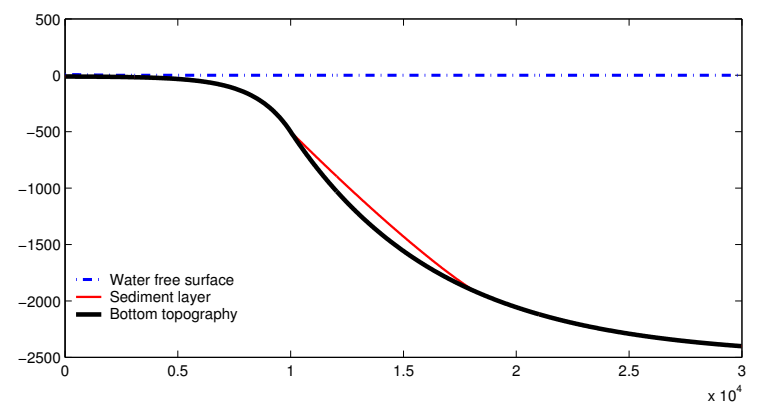

(b) Final configuration of the landslide $(t=600 \mathrm{~s}$.)

Figure 9: Tsunami experiment. (Horizontal $\mathrm{x}(\mathrm{m})$, Vertical $\mathrm{z}(\mathrm{m})$ ) 
that represents most of the tsunami energy. Over the landslide water is sucked, which creates a large trough splitting into two waves, one wave propagating shorewards and the other offshore. Later, the shoreward-propagating wave is followed by a positive wave, responsible for coastal inundation to which attention will be focused. Note that the second positive wave is of about $10 \mathrm{~m}$ height when approaching the coastal line (see Figure 10(f)).

Finally, Figure 9(b) shows the final position of the landslide. The results that we obtain are similar to those descrived by Heinrich et al. in [24].

\section{Conclusions}

In this paper we have introduced a new model to study submarine avalanches and generated tsunamis. The presented model is a two-layer shallow water model including a Coulomb friction type term for the grain layer (Savage-Hutter model for the second layer). It is presented in local coordinates, by taking into account the curvature of a non-erodible bottom over which the avalanche and the tsunami flow. Some of the properties of the model are: the rank of the stationary solutions verifies exactly a dissipation entropy inequality, and the solutions of the model are solutions of Euler equations with hydrostatic pressure. The final system can be rewritten as a hyperbolic system with non-conservative products. We compare the model with that proposed by Heinrich et al., which is an uncoupled model, that mixes local coordinates for the evolution of the grain layer with non local coordinates for the evolution of the fluid layer. We see how the momentum equation for the grain layer of the Heinrich model is obtained under the assumption that the water surface is flat (rigid lid assumption). This model does not verify the properties of our model.

We also present a numerical solver of finite volume type, based on a Roe method for hyperbolic systems with non-conservative products. With a special treatment of the Coulomb term, splitting the discretization of this term into an upwind explicit treatment and a second step to introduce an implicit centered discretization. This allows us to obtain a well-balanced solver for a wide rank of stationary solutions, when the angle of the slope of the grain layer is smaller than the corresponding angle of repose.

The resulting model has been able to simulate sub-aerial and submarine avalanches and the generated tsunami by taking into account the interaction between the flowing mass and the surrounding water.

\section{Appendix: Change of variable}

In this Appendix, we perform the change of variable of Euler equations, from Cartesian coordinates $\vec{X}=(x, z)$ to local coordinates $(X, Z)$ (see Figure 11). We consider that the coordinate $Z$ gives the position of an interior point $\vec{X}$ to the bed, measured in the normal direction to the bed. Thus

$$
0<Z<S(t, x), \quad \text { with } \quad S(t, x)=h_{1}(t, X)+h_{2}(t, X) .
$$

Then, the relation between the Cartesian coordinates $\vec{X}$ and the coordinates $(\bar{x}, Z)$ related to the bed is

$$
\vec{X}=(\bar{x}-Z \sin \theta(\bar{x}), b(\bar{x})+Z \cos \theta(\bar{x})),
$$

where $(\bar{x}, b(\bar{x}))$ is a point of the bed. $\bar{x}$ is the $\mathrm{x}$-Cartesian coordinate of the point $(X, 0)$ (see Figure 11). We also consider a local variable $X(x)$ measuring the arc length along the bed. We will denote by $\nabla_{\vec{X}}$ and $\operatorname{div}_{\vec{X}}$ the gradient and divergence operators in Cartesian coordinates.

We consider the equations of conservation of mass and momentum in $(x, z)$ Cartesian coordinates as follows

$$
\vec{V}=\left(\begin{array}{c}
u \\
v
\end{array}\right), \quad \nabla \cdot \vec{V}=0
$$




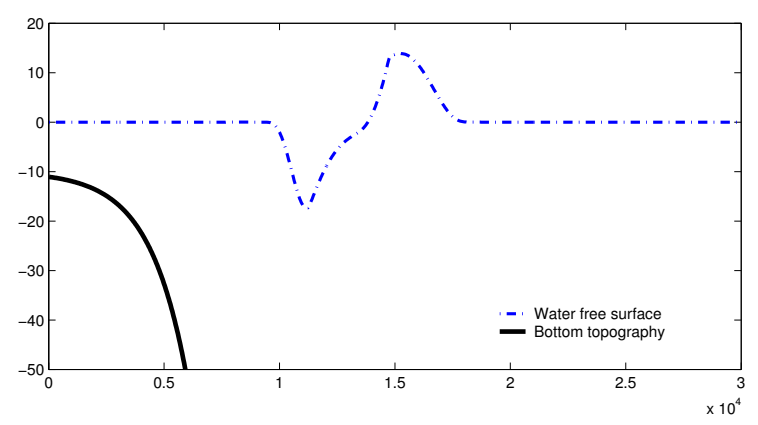

(a) $30 \mathrm{~s}$.

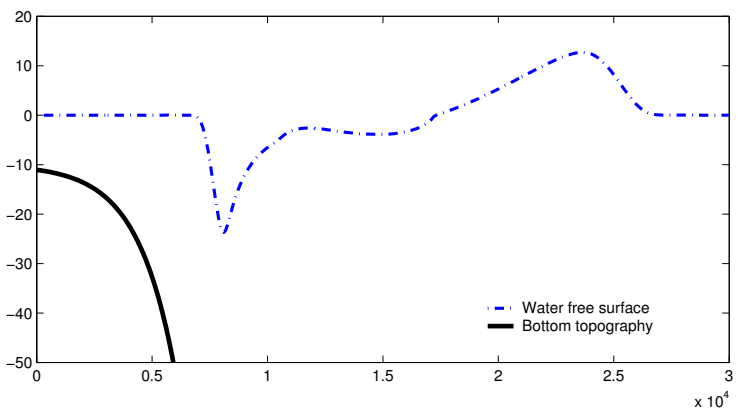

(c) $90 \mathrm{~s}$

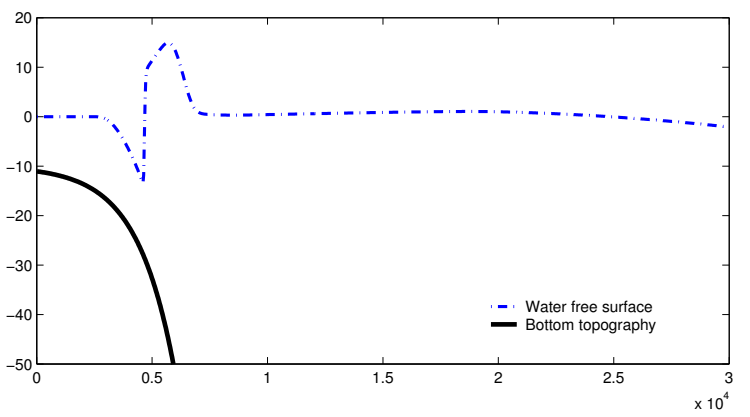

(e) $300 \mathrm{~s}$

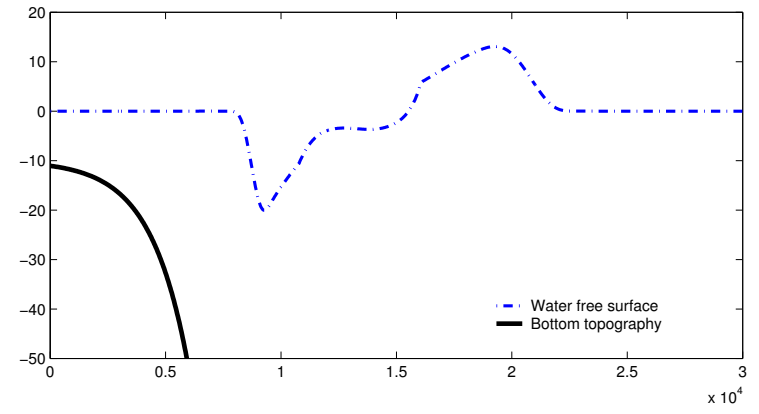

(b) $60 \mathrm{~s}$.

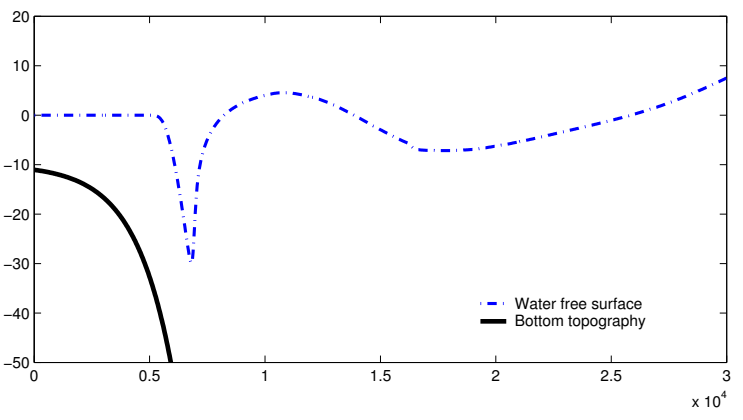

(d) $150 \mathrm{~s}$

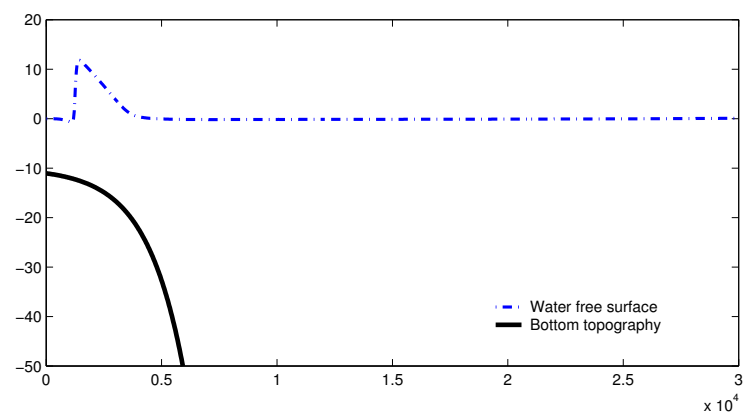

(f) $480 \mathrm{~s}$

Figure 10: Tsunami evolution (zoom). (Horizontal x(m), Vertical $\mathrm{z}(\mathrm{m})$ ) 


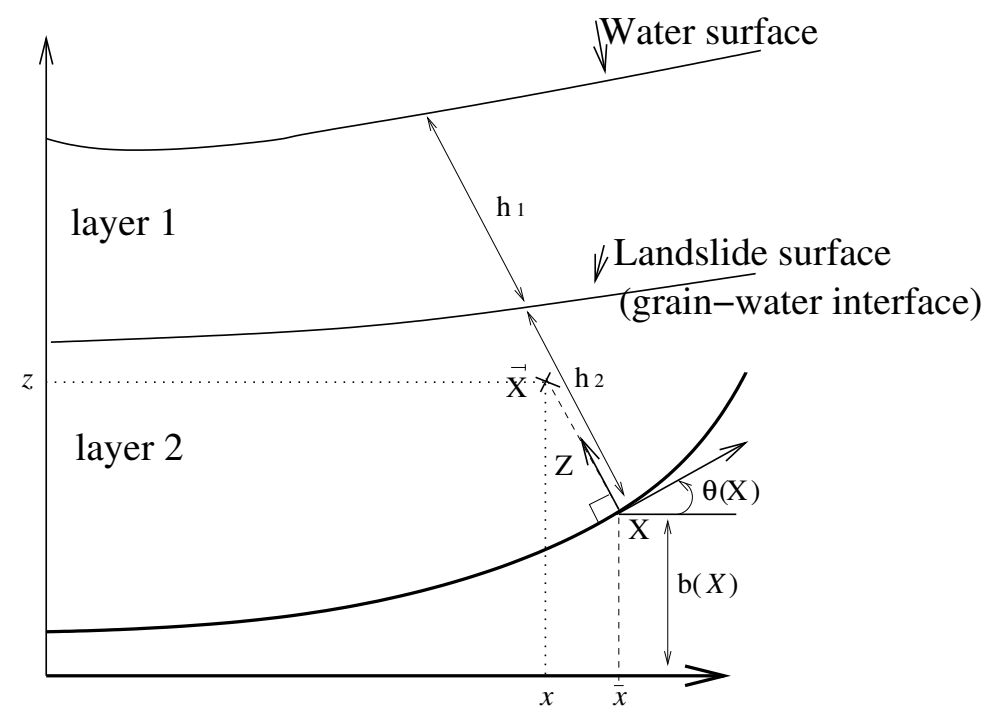

Figure 11: Local coordinates.

$$
\partial_{t}(\rho \vec{V})+\rho \vec{V} \nabla_{\vec{X}} \vec{V}=-\nabla \cdot P+\rho \nabla_{\vec{X}}(\vec{g} \cdot \vec{X}),
$$

where $\vec{g}=(0,-g), g$ being the constant acceleration of gravity. Moreover by $P$ we denote the matrix of constraints,

$$
P=\left(\begin{array}{ll}
p_{x x} & p_{x z} \\
p_{z x} & p_{z z}
\end{array}\right)
$$

(with $p_{x z}=p_{z x}$ ).

From (93) we have

$$
\nabla_{(X, Z)} \vec{X}=\left(\begin{array}{cc}
J \cos \theta & -\sin \theta \\
J \sin \theta & \cos \theta
\end{array}\right), \quad J=1-Z d_{X} \theta
$$

Therefore,

$$
\nabla_{\vec{X}}(X, Z)=\frac{1}{J}\left(\begin{array}{cc}
\cos \theta & \sin \theta \\
-J \sin \theta & J \cos \theta
\end{array}\right) .
$$

The following result will be used in this Appendix:

Lemma: Using the classical chain rule we have

$$
\operatorname{div}_{(X, Z)}\left(J \nabla_{\vec{X}}(X, Z) \vec{V}\right)=J \operatorname{div}_{\vec{X}} \vec{V}, \quad J=\operatorname{det}\left(\nabla_{(X, Z)} \vec{X}\right),
$$

and

$$
\nabla_{\vec{X}} P=\left(\nabla_{\vec{X}}(X, Z)\right)^{T} \nabla_{(X, Z)} P \quad \text { or } \quad\left(\nabla_{\vec{X}} P\right)^{T}=\left(\nabla_{(X, Z)} P\right)^{T} \nabla_{\vec{X}}(X, Z) .
$$

We will also use the following definitions:

$$
\left(\begin{array}{c}
U \\
W
\end{array}\right)=\left(\begin{array}{cc}
\cos \theta & \sin \theta \\
-\sin \theta & \cos \theta
\end{array}\right) \vec{V} .
$$

and

$$
\mathcal{P}=\left(\begin{array}{cc}
\cos \theta & \sin \theta \\
-\sin \theta & \cos \theta
\end{array}\right) P\left(\begin{array}{cc}
\cos \theta & -\sin \theta \\
\sin \theta & \cos \theta
\end{array}\right)=\left(\begin{array}{cc}
\mathcal{P}_{X X} & \mathcal{P}_{X Z} \\
\mathcal{P}_{Z X} & \mathcal{P}_{Z Z}
\end{array}\right) .
$$

As $p_{x z}=p_{x z}$ then $\mathcal{P}_{X Z}=\mathcal{P}_{Z X}$. 


\section{Continuity equation (conservation of mass)}

In an incompressible material the velocity field $\vec{V}$ is solenoidal; so (94) holds. Multiplying (94) by $J$ and using (96) we have

$$
\begin{gathered}
0=J \operatorname{div}_{\vec{X}} \vec{V}=\operatorname{div}_{(X, Z)}\left(J \nabla_{\vec{X}}(X, Z) \vec{V}\right)= \\
=\operatorname{div}_{(X, Z)}\left(\left(\begin{array}{cc}
\cos \theta & \sin \theta \\
-J \sin \theta & J \cos \theta
\end{array}\right) \vec{V}\right)=\operatorname{div}_{(X, Z)}\left(\begin{array}{c}
U \\
J W
\end{array}\right) .
\end{gathered}
$$

Thus, we have the equation,

$$
\partial_{X}(U)+\partial_{Z}(J W)=0 .
$$

This correspond to the first equation of (4)

\section{Conservation of momentum}

In this section, we first find the equation for $U$ and then for $W$.

Equation for U. We add the first component of equation (95) multiplied by $\cos \theta$ to the second component of equation (95) multiplied by $\sin \theta$. Then, we obtain

$$
\begin{gathered}
\rho \partial_{t} U+\rho \operatorname{div}_{\vec{X}}(U \vec{V})+\rho\left(\nabla_{\vec{X}}(\vec{g} \cdot \vec{X})\right)^{T}\left(\begin{array}{c}
\cos \theta \\
\sin \theta
\end{array}\right)= \\
-\operatorname{div}_{\vec{X}}\left(P\left(\begin{array}{c}
\cos \theta \\
\sin \theta
\end{array}\right)\right)+\rho W \operatorname{div}_{\vec{X}}(\theta \vec{V})+\left(\nabla_{\vec{X}} \theta\right)^{T}\left(P\left(\begin{array}{c}
-\sin \theta \\
\cos \theta
\end{array}\right)\right) .
\end{gathered}
$$

In order to apply the rules (96) and (97) we rewrite this last equation as

$$
\begin{gathered}
\rho J \partial_{t} U+\rho J \operatorname{div}_{\vec{X}}\left(\begin{array}{c}
U u \\
U v
\end{array}\right)+J \rho\left(\nabla_{\vec{X}}(\vec{g} \cdot \vec{X})\right)^{T}\left(\begin{array}{c}
\cos \theta \\
\sin \theta
\end{array}\right)= \\
-J \operatorname{div}_{\vec{X}}\left(P\left(\begin{array}{c}
\cos \theta \\
\sin \theta
\end{array}\right)\right)+\rho J W \operatorname{div}_{\vec{X}}(\theta \vec{V})+J\left(\nabla_{\vec{X}} \cdot \theta\right)^{T} P\left(\begin{array}{c}
-\sin \theta \\
\cos \theta
\end{array}\right) .
\end{gathered}
$$

Then, using the rules (96) and (97), we obtain

$$
\begin{gathered}
\rho \partial_{t}(J U)+\rho \operatorname{div}_{(X, Z)}\left(\begin{array}{c}
U^{2} \\
J U W
\end{array}\right)+\rho\left(\nabla_{X, Z}(\vec{g} \cdot \vec{X})\right)^{T}\left(\begin{array}{c}
1 \\
0
\end{array}\right)= \\
=-\operatorname{div}_{(X, Z)}\left(\left(\begin{array}{cc}
1 & 0 \\
0 & J
\end{array}\right)\left(\begin{array}{cc}
\cos \theta & \sin \theta \\
-\sin \theta & \cos \theta
\end{array}\right) P\left(\begin{array}{c}
\cos \theta \\
\sin \theta
\end{array}\right)\right)+ \\
+\rho W \operatorname{div}_{(X, Z)}\left(\begin{array}{c}
\theta U \\
J \theta W
\end{array}\right)+ \\
+\left(\nabla_{(X, Z)} \theta\right)^{T}\left(\begin{array}{ll}
1 & 0 \\
0 & J
\end{array}\right)\left(\begin{array}{cc}
\cos \theta & \sin \theta \\
-\sin \theta & \cos \theta
\end{array}\right) P\left(\begin{array}{c}
-\sin \theta \\
\cos \theta
\end{array}\right) .
\end{gathered}
$$

This correspond to the second equation of (4).

Equation for W: Now, multiplying the first equation of (95) by $(-\sin \theta)$ and the second one by $(\cos \theta)$ and adding them, we obtain

$$
\rho \partial_{t} W+\rho \operatorname{div}_{\vec{X}}(W \vec{V})+\rho\left(\nabla_{\vec{X}}(\vec{g} \cdot \vec{X})\right)^{T}\left(\begin{array}{c}
-\sin \theta \\
\cos \theta
\end{array}\right)=
$$




$$
-\operatorname{div}_{\vec{X}}\left(P\left(\begin{array}{c}
-\sin \theta \\
\cos \theta
\end{array}\right)\right)-\rho U \operatorname{div}_{\vec{X}}(\theta \vec{V})-\left(\nabla_{\vec{X}} \theta\right)^{T}\left(P\left(\begin{array}{c}
\cos \theta \\
\sin \theta
\end{array}\right)\right) .
$$

In order to apply the rules (96) and (97), we rewrite this last equation as

$$
\begin{gathered}
\rho \partial_{t}(J W)+J \rho \operatorname{div}_{\vec{X}}\left(\begin{array}{c}
W u \\
W v
\end{array}\right)+J \rho\left(\nabla_{\vec{X}}(\vec{g} \cdot \vec{X})\right)^{T}\left(\begin{array}{c}
-\sin \theta \\
\cos \theta
\end{array}\right)= \\
-J \operatorname{div}_{\vec{X}}\left(P\left(\begin{array}{c}
-\sin \theta \\
\cos \theta
\end{array}\right)\right)-\rho J U \operatorname{div}_{\vec{X}}(\theta \vec{V})-J\left(\nabla_{\vec{X}} \theta\right)^{T} P\left(\begin{array}{c}
\cos \theta \\
\sin \theta
\end{array}\right) .
\end{gathered}
$$

Then, using the rules (96) and (97), we obtain

$$
\begin{gathered}
\rho \partial_{t}(J W)+\rho \operatorname{div}_{(X, Z)}\left(\begin{array}{c}
W U \\
J W^{2}
\end{array}\right)+\rho\left(\nabla_{X, Z}(\vec{g} \cdot \vec{X})\right)^{T}\left(\begin{array}{c}
0 \\
J
\end{array}\right)= \\
=-\operatorname{div}_{(X, Z)}\left(\left(\begin{array}{cc}
1 & 0 \\
0 & J
\end{array}\right)\left(\begin{array}{cc}
\cos \theta & \sin \theta \\
-\sin \theta & \cos \theta
\end{array}\right) P\left(\begin{array}{c}
-\sin \theta \\
\cos \theta
\end{array}\right)\right)- \\
-\rho U \operatorname{div}_{(X, Z)}\left(\begin{array}{c}
\theta U \\
J \theta W
\end{array}\right)- \\
-\left(\nabla_{(X, Z)} \theta\right)^{T}\left(\begin{array}{cc}
1 & 0 \\
0 & J
\end{array}\right)\left(\begin{array}{cc}
\cos \theta & \sin \theta \\
-\sin \theta & \cos \theta
\end{array}\right) P\left(\begin{array}{c}
\cos \theta \\
\sin \theta
\end{array}\right) .
\end{gathered}
$$

This correspond to the third equation of (4).

\section{Acknowledgments.}

We thank Lev Tsimring, Nicolas Ledante, Dmitri Volfson and Carlos Parés for interesting discussions on the application of the model. We also thank Anael Lemaitre by his interesting discussion about the definition of the stress tensor for the grain-fluid mixture layer. We thank Kolumban Hutter and the other anonymous reviewer for their delaited comments that has helped to improve the paper.

This research has been partially supported by the Spanish Government Research projects MTM2006-08075 and MTM2006-01275. By the ACI Nouvelles Interface de Mathematiques (CNRS), ACI Jeunes Chercheurs (CNRS), ANR Blanche, BLAN-06-1-140039, ACI NIM contract no 03318 and the ANR contract no ANR-06-BLAN-0414, ACI jeunes chercheurs "Analyses mathématiques de paramétrisations en océanographie", Project région Rhône-Alpes "Modélisation d'avalanches".

\section{References}

[1] C. Ancey, Plasticity and geophysical flows: A review J. Non-Newtonian Fluid Mech (2006).

[2] A. Aradian, E. Raphael, P.G. de Gennes, Surface flow of granular materials: A short introduction to some recent models. C.R. Physique 3, 187-196 (2002).

[3] I.S. Aranson, L. S. Tsimring, Continuum theory of partially fluidized granular flows. Phys. Rev. E. vol. 65, 061303 (2002).

[4] G.S. Beaves, D.D. Joseph, Boundary conditions at a naturally permeable wall, J. Fluid Mech., 30, part I, 197-207 (1967).

[5] F. Bouchut, E.D. Fernández-Nieto, A. Mangeney, P.Y. Lagree On new erosion models of Savage-Hutter type for avalanches. Preprint (2007). 
[6] F. Bouchut, A. Mangeney-Castelnau, B. Perthame, J.P Vilotte, A new model of Saint Venant and Savage-Hutter type for gravity driven shallow flows. C.R. Acad. Sci. Paris, Ser I 336 531-536 (2003).

[7] F. Bouchut, M. Westdickenberg, Gravity driven shallow water model for arbitrary topography. Comm. Math. Sci. 2(3), 359-389 (2004).

[8] C. Cassar, M. Nicolas, O. Pouliquen, Submarine granular flows down inclined planes. Physics of Fluids, 17, 103301, DOI 10.1063/1.2069864, (2005).

[9] M.J. Castro, T. Chacón, E.D. Fernández-Nieto, C. Parés On well-balanced finite volume methods for non-conservative non-homogeneous systems. SIAM J. Sci. Comput. 29(3): 10931126, (2007).

[10] M.J. Castro, A.M. Ferreiro, J.A. García, J.M. González, J. Macías, C. Parés, M.E. Vázquez, On the numerical treatment of wet/dry fronts in shallow flows: application to one-layer and two-layer systems. Match Comp. Model. 42 (3-4): 419-439 (2005).

[11] M.J. Castro, J.A. García, J.M. González and C. Parés, A parallel 2d finite volume scheme for solving systems of balance laws with nonconservative products: application to shallow flows. Comp. Meth. Appl. Mech. Eng., 196, 2788-2815 (2006).

[12] T. Chacón, A. Domínguez, E.D. Fernández. A family of stable numerical solvers for Shallow Water equations with source terms. Comp. Meth. Appl. Mech. Eng. 192: 203-225, 2003.

[13] T. Chacón, A. Domínguez, E.D. Fernández. Asymptotically balanced schemes for non-homoegeneous hyperbolic systems - application to the Shallow Water equations. C.R. Acad. Sci. Paris, Ser. I 338: 85-90, 2004.

[14] G. Dal Maso, P.G. LeFloch, F. Murat. Definition and weak stability of nonconservative products. J. Math. Pures Appl. 74: 483-548, 1995.

[15] R.A. Dalrymple, S.T. Grilli, J.T. Kirby. Tsunamis and challenges for accurate modeling. Oceanography, 19:142:151, (2006).

[16] B. Di Martino, F. Flori, C. Giacomoni, P. Orenga. Mathematical and Numerical Analysis of a Tsunami Problem. Math. Models and Methods in Apllied Sciences. Vol. 13, p. 1489-1514, (2003).

[17] D. Dutykh and F. Dias. Water waves generated by a moving bottom. In Anjan Kundu, editor, Tsunami and nonlinear waves, Springer-Verlag (Geo Sc.), 38, 64-68, (2007).

[18] D. Dutykh, Modélisation mathématique des Tsunamis. Thèse de doctorat de l'Ecole Normale Supérieure de Cachan, (2007).

[19] I.V. Fine, A.B. Rabinovich, R.E. Thomson, E.A. Kulikov, Numerical Modeling of Tsunami generation by submarine and subaerial landslides. Submarine Landslides and Tsunamis 6988, Kluwer Acadmic Publishers (2003).

[20] D.L. Geroge, R.J. LeVeque, Finite Volume methods and adaptive refinement for global tsunami propagation and local inundation. Science of Tsunami Hazards, 24, 319-329 (2006).

[21] J.M.N.T. Gray, Granular flow in partially filled slowly rotating drums. J. Fluid Mech, 441, $1-29(2001)$.

[22] Ph. Heinrich, A. Mangeney, S. Guibourg, R. Roche, G. Boudon, J.L. Cheminee, Simulation of water waves generated by a potential debris avalanche in Montserrat, Lesser Antilles, Geophys. Res. Lett., 25(19), 3697-3700 (1998). 
[23] Ph. Heinrich, S. Guibourg, A. Mangeney, R. Roche, Numerical modeling of a landslidegenerated tsunami following a potential explosion of the Montserrat volcano, Phys. Chem. Earth, 24(2), 163-168 (1999).

[24] Ph. Heinrich, A. Piatanesi, H. Hébert, Numerical modelling of tsunami generation and propagation from submarine slumps: the 1998 Papua New Guinea event. Geophys. J. Int. 145, 97-11, (2001)

[25] K. Hutter, K. Jöhnk, B. Svendsen, On interfacial transition conditions in two phase gravity flow, Z angew Math Phys. 45 (1994).

[26] R.M. Iverson, R.P. Denlinger, Flow of variably fluidized granular masses across threedimensional terrain. J. of Geoph. Res. 106, B1, 537-552, (2001).

[27] D.V. Khakhar, A.V. Orpe, P. Andresén, J.M. Ottino, Surface flow of granular materials: model and experiments in heap formation. J. Fluid Mech. 441, 225-264 (2001).

[28] Locat, J., and Mienert, J (Eds.), Submarine Mass Movements and Their Consequences, Kluwer Academic Publishers, Dordrecht, 540 pp (2003).

[29] Lucas, A., and Mangeney, A., Mobility and topography effects for large Valles Marineris landslides on Mars, Geophys. Res. Lett., 34, L10201, doi:10.1029/2007GL029835.

[30] A. Mangeney, Ph. Heinrich, R. Roche, G. Boudon, J.L. Cheminee, Modeling of debris avalanche and generated water waves: Application to real and potential events in Montserrat, Phys. Chem. Earth, 25(9-11), 741-745, (2000).

[31] A. Mangeney-Castelnau, J.P. Vilotte, M.O. Bristeau, B. Perthame, F. Bouchut, C. Siomeoni, S. Yerneni, Numerical modeling of avalanches based on Saint Venant equations using a kinetic scheme. J. Geoph. Res. vo. 108, n. B11, 2527 (2003).

[32] A. Mangeney-Castelnau, F. Bouchut, T.P. Vilotte, E. Lajeneusse, A. Aubertin, M. Pirulli, On the use of Saint-Venant equations to simulate the spreading of a granular mass, J. Gephys. Res. 110 (B9), B09103 (2005).

[33] A. Mangeney-Castelnau, F. Bouchut, N. Thomas, J.P. vilotte, M.O. Bristeau, Numerical modelling of self-channeling granular flows and of their leveel channel deposists, J. Geophs. Res.(SE), 112, F2017, 21pp. (2007).

[34] T. Morales de Luna, A Saint Venant model for gravity driven shallow water flows with variable density and compressibility effects. Math. Comput. Modelling 47, 3-4, 436-444 (2008).

[35] N. Nomanbhoy, K. Satake, Generation mechanism of tsunamis from the 1883 Krakatau eruption, Geopys. Res. Lett., 22, 509-512 (1995).

[36] C. Parés, M.J. Castro 2004, On the well-balance property of Roe's method for nonconservative hyperbolic systems. Applications to shallow-water systems. ESAIM: M2AN, 38(5):821852 (2004).

[37] M. Pelanti, F. Bouchut, A. Mangeney, A Roe-Type Scheme for Two-Phase Shallow Granular Flows with Bottom Topography,in press in Math. Model. Numeric. Analy. (ESAIM:M2AN) (2008).

[38] M. Pirulli, M.O. Bristeau, A. Mangeney, A and C. Scavia, The effect of the earth pressure coefficients on the runout of granular material, Environ. Modell. and Soft., 22(10), 14371454 (2007). 
[39] M. Pirulli and A. Mangeney, Result of Back-Analysis of the Propagation of Rock Avalanches as a Function of the Assumed Rheology, Rock Mech. Rock Engng., 41(1), 59-84 (2008).

[40] E. B. Pitman, 1. Le, A two-fluid model for avalanche and debris flows, Phil. Trans. R. Soc. A, 363:1573-1601 (2005).

[41] Pudasaini, S., and Hutter, K., Avalanche Dynamics, Springer-Verlag, 602p (2007).

[42] Pudasaini, S., Wang Y., Hutter K., Modelling debris flows down general channels. Natural Hazards and Earth System Sciences, 5, 799-819 (2005).

[43] M. Ricchiuto, R. Abgrall, H. Deconinck, Appliation of conservative residual distribution schemes to the solution of the shallow water equations on unstructured meshes. J. Comp. Physics 222, 287-331 (2007)

[44] J. Sander, K. Hutter, Experimental and computational study of channelized water waves generated by a porous body, Acta. Mech., 115, 133-149 (1996).

[45] S.B. Savage, K. Hutter, The dynamics of avalanches of granular materials frominitiation to run-out, Acta Mech. 86, 201-223 (1991).

[46] J.B. Schijf and J.C. Schonfeld, Theoretical considerations on the motion of salt and fresh water, in. Proc. Minn. Int. Hydraulics Conv., joint meeting IAHR Hydro. Div. ASCE., 321-333 (1953).

[47] B. Svendsen, T. Wu, K. Johnk, K. Hutter, On the Role of Mechanical Interactions in the Steady-State Gravity Flow of a Two-Constituent Mixture down an Inclined Plane, Porc. R. Soc. Lond., Vol 452, 1189-1205 (1996).

[48] Vanneste, M., Mienert, J., and Bunz, S., THe Hinlopen Slide: A giant, submarine slope failure on the northern Svalbard margin, Arctic Ocean, Earth Plan. Sci. Lett., 245, 373-388, (2006).

[49] M. Wieland, J.M.N.T. Gray, K. Hutter, Channelized free-surface flow of cohesionless granular avalanches in a chute with shallow lateral curvature. J. Fluid Mech. 392, 73-100 (1999)

[50] T. Wu and K. Hutter, On the Role of the Interface Mechanical Interaction in a GravityDriven Shear Flow of an Ice-Till Mixture. Transport in Porous Media, vol 34, 3-14 (1999)

[51] T. Wu, K. Hutter, B. Svendesen, On shear flow of a satured ice-sediment mixture with thermodynamic equilibrium pressure and momentum exchange. Proc. R. Soc. Lond., A454, 71-88 (1998) 